\title{
Derivations of the Moyal Algebra and Noncommutative Gauge Theories ${ }^{\star}$
}

Jean-Christophe WALLET

Laboratoire de Physique Théorique, Bât. 210, CNRS, Université Paris-Sud 11, F-91405 Orsay Cedex, France

E-mail: jean-christophe.wallet@th.u-psud.fr

Received October 29, 2008, in final form January 17, 2009; Published online January 30, 2009

doi:10.3842/SIGMA.2009.013

\begin{abstract}
The differential calculus based on the derivations of an associative algebra underlies most of the noncommutative field theories considered so far. We review the essential properties of this framework and the main features of noncommutative connections in the case of non graded associative unital algebras with involution. We extend this framework to the case of $\mathbb{Z}_{2}$-graded unital involutive algebras. We show, in the case of the Moyal algebra or some related $\mathbb{Z}_{2}$-graded version of it, that the derivation based differential calculus is a suitable framework to construct Yang-Mills-Higgs type models on Moyal (or related) algebras, the covariant coordinates having in particular a natural interpretation as Higgs fields. We also exhibit, in one situation, a link between the renormalisable $\mathrm{NC} \varphi^{4}$-model with harmonic term and a gauge theory model. Some possible consequences of this are briefly discussed.
\end{abstract}

Key words: noncommutative geometry; noncommutative gauge theories

2000 Mathematics Subject Classification: 81T75; $81 \mathrm{~T} 13$

\section{Introduction}

A class of noncommutative (NC) field theories [1,2] (for general reviews on noncommutative geometry, see [3, 4]) came under increasing scrutiny after 1998 when it was argued $[5,6]$ that string theory might have effective regimes related to noncommutative field theories (NCFT) defined on a NC version of flat 4-dimensional space. This latter is the Moyal space (see e.g. [7, 8]) which has constant commutators between space coordinates. It was further noticed $[9,10]$ that the simplest NC $\varphi^{4}$ model, ( $\varphi$ real-valued) on the 4-dimensional Moyal space is not renormalisable due to the Ultraviolet/Infrared (UV/IR) mixing [9, 10, 11]. This phenomenon stems from the existence of nonplanar diagrams that are UV finite but nevertheless develop IR singularities which when inserted into higher order diagrams are not of renormalisable type [1,2]. A solution to this problem was proposed in 2004 [12, 13]. It amounts to supplement the initial action with a simple harmonic oscillator term leading to a fully renormalisable NCFT (for recent reviews, see e.g. $[14,15])$. This result seems to be related to the covariance of the model under the so called Langmann-Szabo duality [16]. Other renormalisable NC matter field theories have then been identified $[17,18,19,20]$ and studies of the properties of their renormalisation group flows have been carried out [21, 22, 23].

So far, the construction of a renormalisable gauge theory on 4- $D$ Moyal spaces remains a problem. The naive NC version of the Yang-Mills action has UV/IR mixing, which stems from the occurrence of an IR singularity in the polarisation tensor. From a standard one-loop

${ }^{\star}$ This paper is a contribution to the Proceedings of the XVIIth International Colloquium on Integrable Systems and Quantum Symmetries (June 19-22, 2008, Prague, Czech Republic). The full collection is available at http://www.emis.de/journals/SIGMA/ISQS2008.html 
calculation, we easily infer that

$$
\omega_{\mu \nu}(p) \sim(D-2) \Gamma\left(\frac{D}{2}\right) \frac{\tilde{p}_{\mu} \tilde{p}_{\nu}}{\pi^{D / 2}\left(\tilde{p}^{2}\right)^{D / 2}}+\cdots, \quad p \rightarrow 0,
$$

where $\tilde{p}_{\mu} \equiv \Theta_{\mu \nu} p_{\nu}, \Theta_{\mu \nu}$ is the symplectic matrix of the 4-D Moyal algebra and $\Gamma(z)$ denotes the Euler function. This singularity, albeit transverse in the sense of the Slavnov-Taylor-Ward identities, does not correspond to some gauge invariant term. This implies that the recent alternative solution to the UV/IR mixing proposed for the NC $\varphi^{4}$ model in [24], cannot be extended straighforwardly (if possible at all) to the gauge theories. Note that an attempt to reach this goal has been proposed in [25]. It amounts to add to the naive Yang-Mills action a counterterm which remains invariant under a defined BRST symmetry but however can be viewed as a formal infinite series in the gauge potential $A_{\mu}$. The actual effect of this counterterm on the UV/IR mixing of the modified gauge action is still unclear and deserves further investigations.

Recently, an extension of the harmonic solution to the case of gauge theories has been proposed in [26] and [27] (for various reviews, see [28, 15, 29]). These works have singled out, as potential candidate for renormalisable gauge theory on $4-D$ Moyal space, the following generic action

$$
S=\int d^{4} x\left(\frac{1}{4} F_{\mu \nu} \star F_{\mu \nu}+\frac{\Omega^{2}}{4}\left\{\mathcal{A}_{\mu}, \mathcal{A}_{\nu}\right\}_{\star}^{2}+\kappa \mathcal{A}_{\mu} \star \mathcal{A}_{\mu}\right),
$$

where $F_{\mu \nu}=\partial_{\mu} A_{\nu}-\partial_{\nu} A_{\mu}-i\left[A_{\mu}, A_{\nu}\right]_{\star}, A_{\mu}$ is the gauge potential, $\star$ denotes the associative product on the Moyal algebra, $[a, b]_{\star} \equiv a \star b-b \star a,\{a, b\}_{\star} \equiv a \star b+b \star a$ and $\mathcal{A}_{\mu}$ is the covariant coordinates, a natural gauge covariant tensorial form stemming from the existence of a canonical gauge invariant connection in the present $\mathrm{NC}$ framework, as we will recall in a while (see $[26,15])$. The 2 nd and 3rd terms in (1.2) may be viewed as "gauge counterparts" of the harmonic term of [12]. This action has interesting properties [26, 27] deserving further studies. For instance, gauge invariant mass terms for the gauge fields are allowed even in the absence of Higgs mechanism. Besides, the covariant coordinates appear to bear some similarity with Higgs fields. This feature will be examined more closely in the course of the discussion. Unfortunately, the action (1.2) has a non-trivial vacuum which complicates the study of its actual renormalisability [30]. Notice that non trivial vacuum configurations also occur within NC scalar models with harmonic term as shown in [31].

The relevant algebraic framework describing (most of) the classical features of the NC actions considered so far is provided by the differential calculus based on the derivations. This framework has been established in [32, 33, 34] and [35]. For an exhaustive review, see [36] and references therein. The derivation based differential calculus underlies the first prototypes of NC (matrixvalued) field theories [37, 38, 39]. For a review, see [40]. As far as the Moyal spaces are concerned, the "minimal" derivation-based differential calculus generated by the "spatial derivations" $\partial_{\mu}$ underlies (most of) the works that appeared in the literature, so far. This differential calculus is not unique but can be modified in numerous ways. Among these, a simple modification of the minimal differential calculus has been shown recently to give rise to interesting features [41].

The purpose of this paper is to show that the use of the differential calculus based on the derivations of the Moyal algebra or some related $\mathbb{Z}_{2}$-graded version of it permits one to exhibit interesting features related to gauge theories defined on Moyal space. We show, at least within two non trivial examples, that the derivation based differential calculus is a natural framework to construct Yang-Mills-Higgs type models on Moyal (or related) algebras, the so-called "covariant coordinates" [1, 2] having a natural interpretation as Higgs fields. We also exhibit, in one situation, a link between the renormalisable NC $\varphi^{4}$-model with harmonic term $[12,13]$ and a gauge theory model built from the square of a curvature. Some possible consequences of this are briefly discussed. 
The paper is organised as follows. In Section 2.1 of this paper, we review briefly the main properties of the differential calculus based on the derivations of an associative unital algebra and introduce a definition of a $\mathrm{NC}$ connection on a module over the algebra, as a natural generalisation of ordinary connections. The specific properties and simplifications occurring when the module is equal to the algebra, which is the case relevant for the NCFT, are detailed in Section 2.2. In Section 2.3, we extend the analysis to $\mathbb{Z}_{2}$-graded algebras. We construct a $\mathbb{Z}_{2}$-graded differential calculus based on the graded derivations of the algebra, set a definition of NC connection, the corresponding curvature and (unitary) gauge transformations. In Section 3, we focus on the Moyal algebra $\mathcal{M}$. We consider the differential calculus based on the maximal subalgebra of the derivations of $\mathcal{M}$ whose elements can be related to (infinitesimal) symplectomorphisms. Then, a direct application of the results of Section 2.2 leads to a natural construction of Yang-Mills-Higgs models defined on $\mathcal{M}$ where the "covariant coordinates" used in the physics literature can be naturally interpreted as Higgs fields, due to the existence of a gauge invariant canonical connection. In Section 4 we compare in detail the salient mathematical features underlying the NC differential calculus of Section 3 to those for the NC geometry stemming from the finite dimensional matrix algebra $M_{n}(\mathbb{C})$ as well as for the algebra of matrix valued functions $C^{\infty}(M) \otimes M_{n}(\mathbb{C})$. In some sense, the case considered in Section 3 interpolates between these two latter situations. The classical properties of the NC Yang-Mills-Higgs actions are also analysed. Explicit one-loop computation of the vacuum polarisation tensor shows that this latter still exhibits an IR singularity of the type given in (1.1). In Section 5 we consider a $\mathbb{Z}_{2}$-graded version of the Moyal algebra $\mathcal{M}$ built from two copies of $\mathcal{M}$ and apply the general results derived in Section 2.3 to the construction of a differential calculus generated by a $\mathbb{Z}_{2^{-}}$ graded extension of the derivation algebra considered in Section 3. We show that the gauge theory action built from the "square" of the resulting curvature involves as contributions both the action (1.2) derived in [26] and [27] as well the renormalisable NC $\varphi^{4}$-model with harmonic term elaborated in $[12,13]$, therefore exhibiting a link between this latter renormalisable NC scalar theory and gauge theories. Finally, a summary of all the main results is presented in Section 6.

\section{Differential calculus based on derivations}

\subsection{General properties}

Let $\mathbb{A}$ be an associative $*$-algebra with unit $\mathbb{I}$ and center $\mathcal{Z}(\mathbb{A})$. We denote the involution by $a \mapsto a^{\dagger}, \forall a \in \mathbb{A}$. The differential calculus based on the derivations of $\mathbb{A}$ is a natural NC generalisation of the usual de Rham differential calculus on a manifold. Basically, the role of the vector fields is now played by the derivations of the algebra. In this subsection, we collect the main properties that will be used in this paper. More details can be found in [32, 34, 33, 35].

Definition 2.1. The vector space of derivations of $\mathbb{A}$ is the space of linear maps defined by $\operatorname{Der}(\mathbb{A})=\{X: \mathbb{A} \rightarrow \mathbb{A} / X(a b)=X(a) b+a X(b), \forall a, b \in \mathbb{A}\}$. The derivation $X \in \operatorname{Der}(\mathbb{A})$ is called real if $(X(a))^{\dagger}=X\left(a^{\dagger}\right), \forall a \in \mathbb{A}$.

The essential properties of the spaces of derivations of $\mathbb{A}$ can be summarised in the following proposition.

Proposition 2.1. $\operatorname{Der}(\mathbb{A})$ is a $\mathcal{Z}(\mathbb{A})$-module for the product $(f X) a=f(X a), \forall f \in \mathcal{Z}(\mathbb{A})$, $\forall X \in \operatorname{Der}(\mathbb{A})$ and a Lie algebra for the bracket $[X, Y] a=X Y a-Y X a, \forall X, Y \in \operatorname{Der}(\mathbb{A})$. The vector subspace of inner derivations is defined by $\operatorname{Int}(\mathbb{A})=\left\{\operatorname{Ad}_{a}: b \mapsto[a, b] / a \in \mathbb{A}\right\} \subset \operatorname{Der}(\mathbb{A})$. It is a $\mathcal{Z}(\mathbb{A})$-submodule and Lie ideal. The vector subspace of outer derivations is $\operatorname{Out}(\mathbb{A})=$ $\operatorname{Der}(\mathbb{A}) / \operatorname{Int}(\mathbb{A})$. The following canonical short exact sequence of Lie algebras and $\mathcal{Z}(\mathbb{A})$-modules holds: $0 \longrightarrow \operatorname{Int}(\mathbb{A}) \longrightarrow \operatorname{Der}(\mathbb{A}) \longrightarrow \operatorname{Out}(\mathbb{A}) \longrightarrow 0$. 
The main features of the differential calculus based on $\operatorname{Der}(\mathbb{A})$ are involved in the following proposition. Notice that both the Lie algebra structure and the $\mathcal{Z}(\mathbb{A})$-module structures for $\operatorname{Der}(\mathbb{A})$ are used as essential ingredients in the construction.

Proposition 2.2. Let $\underline{\Omega}_{\mathrm{Der}}^{n}(\mathbb{A})$ denote the space of $\mathcal{Z}(\mathbb{A})$-multilinear antisymmetric maps from $\operatorname{Der}(\mathbb{A})^{n}$ to $\mathbb{A}$, with $\underline{\Omega}_{\operatorname{Der}}^{0}(\mathbb{A})=\mathbb{A}$ and let $\underline{\Omega}_{\operatorname{Der}}^{\bullet}(\mathbb{A})=\bigoplus_{n>0} \underline{\Omega}_{\operatorname{Der}}^{n}(\mathbb{A})$. Then $\left(\underline{\Omega}_{\operatorname{Der}}^{\bullet}(\mathbb{A}), \times, \hat{d}\right)$ is a $\mathbb{N}$ graded differential algebra with the product $\times$ on $\underline{\Omega}_{\mathrm{Der}}^{\bullet}(\mathbb{A})$ and differential $\hat{d}: \underline{\Omega}_{\mathrm{Der}}^{n}(\mathbb{A}) \rightarrow \underline{\Omega}_{\mathrm{Der}}^{n+1}(\mathbb{A})$ satisfying $\hat{d}^{2}=0$, respectively defined for $\forall \omega \in \underline{\Omega}_{\text {Der }}^{p}(\mathbb{A}), \eta \in \underline{\Omega}_{\text {Der }}^{q}(\mathbb{A})$ by:

$$
\begin{aligned}
(\omega \times \eta)\left(X_{1}, \ldots, X_{p+q}\right)= & \frac{1}{p ! q !} \sum_{\sigma \in \mathfrak{S}_{p+q}}(-1)^{\operatorname{sign}(\sigma)} \omega\left(X_{\sigma(1)}, \ldots, X_{\sigma(p)}\right) \eta\left(X_{\sigma(p+1)}, \ldots, X_{\sigma(p+q)}\right), \\
\hat{d} \omega\left(X_{1}, \ldots, X_{p+1}\right)= & \sum_{i=1}^{p+1}(-1)^{i+1} X_{i} \omega\left(X_{1}, \cdots \vee_{i} \ldots, X_{p+1}\right) \\
& +\sum_{1 \leq i<j \leq p+1}(-1)^{i+j} \omega\left(\left[X_{i}, X_{j}\right], \cdots \vee_{i} \cdots \vee_{j} \ldots, X_{p+1}\right),
\end{aligned}
$$

where $\sigma$ denotes permutation and the symbol $\vee_{i}$ indicates that $X_{i}$ is omitted.

It turns out that a differential calculus can also be built from suitable subalgebras of $\operatorname{Der}(\mathbb{A})$. The following proposition holds [32, 33]

Proposition 2.3. Let $\mathcal{G} \subset \operatorname{Der}(\mathbb{A})$ denote a Lie subalgebra which is also a $\mathcal{Z}(\mathbb{A})$-submodule. Then, a restricted derivation-based differential calculus $\underline{\Omega}_{\mathcal{G}}^{\bullet}(\mathbb{A})$ can be built from $\mathcal{G}$. It is obtained from Proposition 2.2 by replacing the set of $\underline{\Omega}_{\text {Der }}^{n}(\mathbb{A}), \forall n \in \mathbb{N}$ by the set of $\mathcal{Z}(\mathbb{A})$-multilinear antisymmetric maps from $\mathcal{G}^{n}$ to $\mathbb{A}$ for $n \geq 0$ and still using (2.1).

From now on, we will not write explicitly the product of forms $\times$. It should be obvious from the context when the relevant type of product is used.

In this paper, we will consider a natural NC generalisation of ordinary connections, as introduced in $[32,34,33]$ to which we refer for more details. It uses left or right finite projective modules on the associative algebra. Notice that alternative NC extensions of connections based on bimodules were considered in [35]. From now on, we denote by $\mathbb{M}$ a right $\mathbb{A}$-module. Let $h: \mathbb{M} \otimes \mathbb{M} \rightarrow \mathbb{A}$ denote a Hermitian structure ${ }^{1}$ on $\mathbb{A}$. The connection, curvature and gauge transformations are given as follows:

Definition 2.2. A $N C$ connection on $\mathbb{M}$ is a linear map $\nabla: \mathbb{M} \times \operatorname{Der}(\mathbb{A}) \rightarrow \mathbb{M}$ satisfying:

$$
\begin{aligned}
& \nabla_{X}(m a)=m X(a)+\nabla_{X}(m) a, \quad \nabla_{f X}(m)=f \nabla_{X}(m), \\
& \nabla_{X+Y}(m)=\nabla_{X}(m)+\nabla_{Y}(m),
\end{aligned}
$$

$\forall X, Y \in \operatorname{Der}(\mathbb{A}), \forall a \in \mathbb{A}, \forall m \in \mathbb{M}, \forall f \in \mathcal{Z}(\mathbb{A})$. A Hermitian NC connection is a NC connection satisfying in addition $X\left(h\left(m_{1}, m_{2}\right)\right)=h\left(\nabla_{X}\left(m_{1}\right), m_{2}\right)+h\left(m_{1}, \nabla_{X}\left(m_{2}\right)\right), \forall m_{1}, m_{2} \in \mathbb{M}$, and for any real derivation in $\operatorname{Der}(\mathbb{A})$. The curvature of $\nabla$ is the linear map $F(X, Y): \mathbb{M} \rightarrow \mathbb{M}$ defined by

$$
F(X, Y) m=\left[\nabla_{X}, \nabla_{Y}\right] m-\nabla_{[X, Y]} m, \quad \forall X, Y \in \operatorname{Der}(\mathbb{A}) .
$$

Definition 2.3. The gauge group of $\mathbb{M}$ is defined as the group of automorphisms of $\mathbb{M}$ as a right $\mathbb{A}$-module.

\footnotetext{
${ }^{1}$ Recall that a Hermitian structure is a sesquilinear map such that $h\left(m_{1}, m_{2}\right)^{\dagger}=h\left(m_{2}, m_{1}\right), h\left(m a_{1}, m a_{2}\right)=$ $a_{1}^{\dagger} h(m 1, m 2) a_{2}, \forall m_{1}, m_{2} \in \mathbb{M}, \forall a_{1}, a_{2} \in \mathbb{A}$.
} 
Proposition 2.4. For any $g$ in the gauge group of $\mathbb{M}$ and for any $N C$ connection $\nabla$, the map $\nabla_{X}^{g}=g^{-1} \circ \nabla_{X} \circ g: \mathbb{M} \rightarrow \mathbb{M}$ defines a NC connection. Then, one has $F(X, Y)^{g}=$ $g^{-1} \circ F(X, Y) \circ g$.

It is convenient to require that the gauge transformations are compatible with the Hermitian structure, that is $h\left(g\left(m_{1}\right), g\left(m_{2}\right)\right)=h\left(m_{1}, m_{2}\right)$. This defines a NC analogue of unitary gauge transformations. From now on, we will only consider unitary gauge transformations.

\subsection{The free module case}

In the special case where $\mathbb{M}=\mathbb{A}$, that will be the case of interest for the ensuing discussion, additional simplifications occur. It is further convenient to choose the canonical Hermitian structure $h_{0}\left(a_{1}, a_{2}\right)=a_{1}^{\dagger} a_{2}$.

Proposition 2.5. Assume that $\mathbb{M}=\mathbb{A}$ and $h_{0}\left(a_{1}, a_{2}\right)=h\left(a_{1}, a_{2}\right)=a_{1}^{\dagger} a_{2}$. Then:

i) Any NC connection is entirely determined by $\nabla_{X}(\mathbb{I})$ via $\nabla_{X}(a)=\nabla_{X}(\mathbb{I}) a+X(a), \forall X \in$ $\operatorname{Der}(\mathbb{A}), \forall a \in \mathbb{A}$. The 1 -form connection $A \in \underline{\Omega}_{\mathrm{Der}}^{1}(\mathbb{A})$ is defined by $A: X \rightarrow A(X)=$ $\nabla_{X}(\mathbb{I}), \forall X \in \operatorname{Der}(\mathbb{A})$.

ii) Assume that the derivations $X$ are real. Then, a NC connection is Hermitian when $\nabla_{X}(\mathbb{I})^{\dagger}=-\nabla_{X}(\mathbb{I})$.

iii) The gauge group can be identified with the group of unitary elements of $\mathbb{A}, \mathcal{U}(\mathbb{A})$ and one has $\nabla_{X}(\mathbb{I})^{g}=g^{\dagger} \nabla_{X}(\mathbb{I}) g+g^{\dagger} X(g), F(X, Y)^{g}=g^{\dagger} F(X, Y) g, \forall X, Y \in \operatorname{Der}(\mathbb{A}), \forall a \in \mathbb{A}$.

Proof. $i$ ) follows directly from Definition 2.2 (set $m=\mathbb{I}$ in the 1st of (2.2)). Note that the relation $\nabla_{X}(a)=\nabla_{X}(\mathbb{I}) a+X(a)$ can be reobtained from the map $\nabla: \underline{\Omega}_{\text {Der }}^{0}(\mathbb{A}) \rightarrow \underline{\Omega}_{\text {Der }}^{1}(\mathbb{A}), \nabla(a)=$ $A a+\hat{d} a$ with $A: X \rightarrow A(X)=\nabla_{X}(\mathbb{I})$. For $\left.i i\right)$, one has $\left(\nabla_{X}\left(a_{1}\right)^{\dagger} a_{2}+a_{1}^{\dagger} \nabla_{X}\left(a_{2}\right)=X\left(a_{1}^{\dagger} a_{2}\right)+\right.$ $a_{1}^{\dagger}\left(\nabla_{X}(\mathbb{I})^{\dagger}+\nabla_{X}(\mathbb{I})\right) a_{2}$ where the last equality stems from the expression for $\nabla_{X}(a)$ given in $\left.i\right)$ and the fact that $X$ is assumed to be real. From this follows ii). For iii), use Definition 2.3 and compatibility of gauge transformations with $h_{0}$ which gives $g(a)=g(\mathbb{I}) a$ and $h_{0}\left(g\left(a_{1}\right), g\left(a_{2}\right)\right)=$ $a_{1}^{\dagger} g(\mathbb{I})^{\dagger} g(\mathbb{I}) a_{2}=h_{0}\left(a_{1}, a_{2}\right)$. Then, the gauge transformations for $\nabla_{X}(\mathbb{I})$ and the curvature stems from Proposition 2.4, the expression for $\nabla_{X}(a)$ in $\left.i\right)$ and the expression for $F(X, Y)$.

Definition 2.4. A tensor 1-form is a 1-form having the following gauge transformations:

$$
\mathcal{A}^{g}=g^{\dagger} \mathcal{A} g, \quad \forall g \in \mathcal{U}(\mathbb{A}) .
$$

There is a special situation where canonical gauge invariant connections can show up, as indicated in the following proposition.

Proposition 2.6. Assume that there exists $\eta \in \Omega_{\mathrm{Der}}^{1}(\mathbb{A})$, such that $\hat{d} a=[\eta, a], \forall a \in \mathbb{A}$. Consider the map $\nabla^{\text {inv }}: \underline{\Omega}_{\text {Der }}^{0}(\mathbb{A}) \rightarrow \underline{\Omega}_{\text {Der }}^{1}(\mathbb{A}), \nabla^{\operatorname{inv}}(a)=\hat{d} a-\eta a, \forall a \in \mathbb{A}$, so that $\nabla_{X}^{\text {inv }}(a)=$ $X(a)-\eta(X) a$. Then, the following properties hold:

i) $\nabla^{\mathrm{inv}}$ defines a connection which is gauge invariant, called the canonical connection.

ii) For any $N C$ connection $\nabla, \mathcal{A} \equiv \nabla-\nabla^{\mathrm{inv}}=A+\eta$ defines a tensor form. $\mathcal{A}(X), \forall X \in$ $\operatorname{Der}(\mathbb{A})$ are called the covariant coordinates of $\nabla$.

Proof. Since any 1-form can serve as defining a connection in view of Proposition 2.5, $\nabla^{\operatorname{inv}}(a)=$ $\hat{d} a-\eta a$ is a connection. Notice that it reduces to $\nabla^{\operatorname{inv}}(a)=-a \eta$, since $\hat{d} a=[\eta, a]$. Then, one has $\left(\nabla^{\mathrm{inv}}\right)^{g}(a)=g^{\dagger} \nabla^{\mathrm{inv}}(g a)=g^{\dagger}(d(g a)-\eta g a)=g^{\dagger}(-g a \eta)=-a \eta=\nabla^{\mathrm{inv}}(a)$, which shows $\left.i\right)$. The property ii) stems simply from Definition 2.4 and the gauge transformations of a NC connection. 
The existence of canonical connections translates into some rather general properties of the curvatures, in particular the curvature for the canonical connection. Gauge theories defined on Moyal spaces are a particular example of this, as shown in the next section.

Lemma 2.1. Let $F^{\text {inv }}(X, Y) \equiv \eta[X, Y]-[\eta(X), \eta(Y)]$ denote the curvature for the canonical connection. Assume again that there exists $\eta \in \Omega_{\text {Der }}^{1}(\mathbb{A})$, such that $\hat{d} a=[\eta, a], \forall a \in \mathbb{A}$. Then, the following properties hold:

i) $F^{\text {inv }}(X, Y) \equiv \eta[X, Y]-[\eta(X), \eta(Y)] \in \mathcal{Z}(\mathbb{A})$.

ii) The curvature of any NC connection defined by the tensor 1-form $\mathcal{A}$ can be written as

$$
F(X, Y)=([\mathcal{A}(X), \mathcal{A}(Y)]-\mathcal{A}[X, Y])-([\eta(X), \eta(Y)]-\eta[X, Y]), \quad \forall X, Y \in \operatorname{Der}(\mathbb{A}) .
$$

Proof. First, from the definition of $\nabla^{\text {inv }}(a)$ in Proposition 2.6, one infers that the 2-form curvature associated to the canonical connection is $F^{\text {inv }}(a) \equiv \nabla^{\operatorname{inv}}\left(\nabla^{\operatorname{inv}}(a)\right)=-(\hat{d} \eta-\eta \eta)(a)$, $\forall a \in \mathbb{A}$. Then, one obtains $F^{\text {inv }}(X, Y)=\eta[X, Y]-[\eta(X), \eta(Y)]$. Then, from $\hat{d} a=[\eta, a]$ and $\hat{d}^{2}=$ 0 , one has $\hat{d}(\hat{d} a)=\hat{d}(\eta a-a \eta)=[\hat{d} \eta, a]-[\eta, \hat{d} a]=[\hat{d} \eta, a]-[\eta,[\eta, a]]=[\hat{d} \eta-\eta \eta, a]$. From this follows the property $i)$. Next, one has $\nabla_{X}(a)=\mathcal{A}(X) a-a \eta(X)$ so that $\left[\nabla_{X}, \nabla_{Y}\right](a)=[\mathcal{A}(X), \mathcal{A}(Y)] a-$ $a[\eta(X), \eta(Y)]$. Therefore $F(X, Y)(a)=([\mathcal{A}(X), \mathcal{A}(Y)]-\mathcal{A}([X, Y])) a-a([\eta(X), \eta(Y)]-\eta([X, Y]))$. This last expression, combined with the property $i$ ) implies $i i)$.

Notice that this proposition will be relevant when considering the NC gauge theories on Moyal algebras. A somewhat similar (but not identical!) situation occurs for the algebra of matrices $M_{n}(\mathbb{C})$ as well as for the algebra of matrix valued functions.

\subsection{Graded differential calculus}

In this subsection, we extend the previous algebraic scheme to the case of differential calculus based on the derivations of a graded algebra. We consider only the $\mathbb{Z}_{2}$-graded case. The extension to graduations based on additive groups as well as to more general structures [43] will be reported in a separate work [44].

To fix the notations, let $\mathbb{A}^{\bullet}$ be a $\mathbb{Z}_{2}$-graded associative unital $*$-algebra, namely $\mathbb{A}^{\bullet}=$ $\bigoplus_{\alpha=0,1} \mathbb{A}^{\alpha}$ where $\mathbb{A}^{\alpha}=\left\{a \in \mathbb{A}^{\bullet} /|a|=\alpha\right\},|a|$ denoting the homogeneous degree of $a$, and $\mathbb{A}^{\alpha} \mathbb{A}^{\beta} \subseteq \mathbb{A}^{(\alpha+\beta)(\bmod 2)}$. The graded bracket on $\mathbb{A}^{\bullet}$ is $[a, b] \bullet \equiv a b-(-1)^{|a||b|} b a, \forall a, b \in \mathbb{A}^{\bullet}$. Let $\mathcal{Z}\left(\mathbb{A}^{\bullet}\right)$ denote the center of $\mathbb{A}^{\bullet}$, a graded-commutative algebra $\left(a \in \mathcal{Z}\left(\mathbb{A}^{\bullet}\right) \Longleftrightarrow[a, b] \bullet=0\right.$, $\left.\forall b \in \mathbb{A}^{\bullet}\right)$. Graded modules will be needed in the present framework. Recall that they are graded vector spaces $\mathbb{M}^{\bullet}=\bigoplus_{\alpha=0,1} \mathbb{M}^{\alpha}$ satisfying $\mathbb{M}^{\alpha} \mathbb{A}^{\beta} \subseteq \mathbb{M}^{\alpha+\beta}$ and $\mathbb{A}^{\beta} \mathbb{M}^{\alpha} \subseteq \mathbb{M}^{\alpha+\beta}$ respectively for a right- and left- $\mathbb{A}^{\bullet}$-module.

Definition 2.5. The $\mathbb{Z}_{2}$-graded vector space of derivations of $\mathbb{A}^{\bullet}$ is $\operatorname{Der}\left(\mathbb{A}^{\bullet}\right)=\bigoplus_{\alpha=0,1} \operatorname{Der}^{\alpha}\left(\mathbb{A}^{\bullet}\right)$ where $\operatorname{Der}^{\alpha}\left(\mathbb{A}^{\bullet}\right)$ is the vector space of linear maps $X$ of homogeneous degree $|X|=\alpha$ defined by $\operatorname{Der}^{\alpha}\left(\mathbb{A}^{\bullet}\right)=\left\{X: \mathbb{A}^{\beta} \rightarrow \mathbb{A}^{\alpha+\beta} / X(a b)=X(a) b+(-1)^{\alpha \beta} a X(b), \forall a \in \mathbb{A}^{\beta}, \forall b \in \mathbb{A}^{\bullet}\right\}$.

Most of the properties of the spaces of derivations in the non-graded case can be extended to the graded situation. In the rest of this paper, we will consider the case where the graded module is the graded algebra itself, i.e. $\mathbb{M}^{\bullet}=\mathbb{A}^{\bullet}$.

Proposition 2.7. $\operatorname{Der}\left(\mathbb{A}^{\bullet}\right)$ is a graded right $\mathcal{Z}\left(\mathbb{A}^{\bullet}\right)$-module for the product $(z X)(a)=z(X(a))$, a graded left $\mathcal{Z}\left(\mathbb{A}^{\bullet}\right)$-module for the product $(X z)(a)=(-1)^{|a||z|}(X(a)) z, \forall z \in \mathcal{Z}\left(\mathbb{A}^{\bullet}\right), \forall X \in$ $\operatorname{Der}\left(\mathbb{A}^{\bullet}\right)$ of homogeneous degree and a $\mathbb{Z}_{2}$-graded Lie algebra of the graded bracket $[X, Y] \bullet=$ $X Y-(-1)^{|X||Y|} Y X, \forall X, Y \in \operatorname{Der}\left(\mathbb{A}^{\bullet}\right)$. The $\mathbb{Z}_{2}$-graded vector subspaces of inner derivations is defined by $\operatorname{Int}^{\bullet}(\mathbb{A})=\left\{\operatorname{Ad}_{a}: b \mapsto[a, b] \bullet / a \in \mathbb{A}^{\bullet}\right\} \subset \operatorname{Der} \bullet(\mathbb{A})$. It is a $\mathbb{Z}_{2}$-graded $\mathcal{Z}\left(\mathbb{A}^{\bullet}\right)$ submodules and Lie subalgebra. The $\mathbb{Z}_{2}$-graded vector subspace of outer derivations is defined as $\operatorname{Out}\left(\mathbb{A}^{\bullet}\right)=\operatorname{Der}\left(\mathbb{A}^{\bullet}\right) / \operatorname{Int}\left(\mathbb{A}^{\bullet}\right)$. 
Proof. The properties follow from straightforward calculations.

Definition 2.6. The space $\Omega_{\operatorname{Der}}^{n, \alpha}\left(\mathbb{A}^{\bullet}\right), \alpha \in\{0,1\}, n \in \mathbb{N}$ denotes the vector space of $n$-linear maps $\omega$ with homogenous degree $\alpha, \omega:\left(\operatorname{Der}\left(\mathbb{A}^{\bullet}\right)\right)^{\times n} \rightarrow \mathbb{A}^{\bullet}$ defined by

i) $\Omega_{\operatorname{Der}}^{0, \alpha}\left(\mathbb{A}^{\bullet}\right)=\mathbb{A}^{\alpha}, \forall \alpha \in\{0,1\}$ and, $\forall \omega \in \Omega_{\operatorname{Der}}^{n, \alpha}\left(\mathbb{A}^{\bullet}\right), \forall X_{i} \in \operatorname{Der}\left(\mathbb{A}^{\bullet}\right)$ of homogeneous degree,

ii) $\omega\left(X_{1}, \ldots, X_{n}\right) \in \mathbb{A}^{\alpha+\sum_{l=1}^{n}\left|X_{l}\right|}$,

iii) $\omega\left(X_{1}, \ldots, X_{n} z\right)=\omega\left(X_{1}, \ldots, X_{n}\right) z, \forall z \in \mathcal{Z}\left(\mathbb{A}^{\bullet}\right)$, where $(X z)$ is given in Proposition 2.7,

iv) $\omega\left(X_{1}, X_{2}, \ldots, X_{i}, X_{i+1}, \ldots, X_{n}\right)=(-1)^{\left|X_{i}\right|\left|X_{i+1}\right|} \omega\left(X_{1}, X_{2}, \ldots, X_{i+1}, X_{i}, \ldots, X_{n}\right)$.

Proposition 2.8. Let us define $\Omega_{\operatorname{Der}}\left(\mathbb{A}^{\bullet}\right)=\bigoplus_{n \in \mathbb{N}, \alpha=0,1} \Omega_{\text {Der }}^{n, \alpha}\left(\mathbb{A}^{\bullet}\right)$.

Then, the triplet $\left(\Omega_{\mathrm{Der}}\left(\mathbb{A}^{\bullet}\right), \times, \hat{d}\right)$ is a bigraded differential algebra with the product $\times$ on $\Omega_{\text {Der }}\left(\mathbb{A}^{\bullet}\right)$ and differential $\hat{d}: \Omega_{\operatorname{Der}}^{n, \alpha}\left(\mathbb{A}^{\bullet}\right) \rightarrow \Omega_{\text {Der }}^{n+1, \alpha}\left(\mathbb{A}^{\bullet}\right)$ such that $\hat{d}^{2}=0$ defined by:

i) For any $\omega \in \Omega_{\mathrm{Der}}^{p, \alpha}\left(\mathbb{A}^{\bullet}\right), \eta \in \Omega_{\mathrm{Der}}^{q, \alpha}\left(\mathbb{A}^{\bullet}\right)$

$$
\begin{aligned}
& \omega \times \eta\left(X_{1}, X_{2}, \ldots, X_{p+q}\right) \\
& =\frac{1}{p ! q !} \sum_{\sigma \in \mathfrak{S}(p+q)}(-1)^{\operatorname{sign}(\sigma)}(-1)^{\sum_{k<l, \sigma(k)>\sigma(l)}\left|X_{k}\right|\left|X_{l}\right|+|\eta| \sum_{\sigma(k) \leq p}\left|X_{k}\right|} \\
& \quad \times \omega\left(X_{\sigma(1)}, \ldots, X_{\sigma(p)}\right) \eta\left(X_{\sigma(p+1)}, \ldots, X_{\sigma(p+q)}\right) .
\end{aligned}
$$

ii) The differential is defined, $\forall \omega \in \Omega_{\mathrm{Der}}^{n, \alpha}\left(\mathbb{A}^{\bullet}\right)$ by:

$$
\begin{aligned}
\hat{d} \omega\left(X_{1},\right. & \left.X_{2}, \ldots, X_{n+1}\right) \\
= & \sum_{k=1}^{n+1}(-1)^{k+1}(-1)^{|\omega|\left|X_{k}\right|}(-1)^{\sum_{n=1}^{k-1}\left|X_{k}\right|\left|X_{n}\right|} X_{k} \omega\left(X_{1}, X_{2}, \ldots, \vee_{k}, \ldots, X_{n+1}\right) \\
& +\sum_{\substack{1 \leq k<l \leq n+1\\
}}(-1)^{k+l}(-1)^{\left|X_{k}\right|\left|X_{l}\right|}(-1)^{\sum_{n=1}^{k-1}\left|X_{k}\right|\left|X_{n}\right|} \\
& \quad \times(-1)^{\sum_{n=1}^{l-1}\left|X_{l}\right|\left|X_{n}\right|} \omega\left(\left[X_{k}, X_{l}\right] \bullet, \ldots, \vee_{k}, \cdots \vee_{l}, \ldots, X_{n+1}\right),
\end{aligned}
$$

where the symbol $\vee_{i}$ indicates that $X_{i}$ is omitted.

iii) A restricted differential calculus is obtained as follows. Let $\mathcal{G}^{\bullet} \subset \operatorname{Der}\left(\mathbb{A}^{\bullet}\right)$ denote a $\mathbb{Z}_{2}$ graded Lie subalgebra and $\mathcal{Z}\left(\mathbb{A}^{\bullet}\right)$-submodule. A restricted differential calculus based on $\mathcal{G}^{\bullet}$ can be constructed by replacing in $i$ ) and ii) $\operatorname{Der}\left(\mathbb{A}^{\bullet}\right)$ by $\mathcal{G}^{\bullet}$ and each $\Omega_{\operatorname{Der}}^{n, \alpha}\left(\mathbb{A}^{\bullet}\right), \alpha \in\{0,1\}$, $n \in \mathbb{N}$ by the vector space of $n$-linear maps $\omega$ with homogenous degree $\alpha, \omega:\left(\mathcal{G}^{\bullet}\right)^{\times n} \rightarrow \mathbb{A}^{\bullet}$.

Proof. The proposition can be verified from direct calculation.

As in Section 2.2, we will not write explicitly the symbol $\times$ for the product of forms as the product to be used in each case is obvious from the context.

We now define the connection on $\mathbb{A}^{\bullet}$ and its curvature as follows:

Definition 2.7. Let $\Omega_{\text {Der }}^{n, \bullet} \equiv \bigoplus_{\alpha=0,1} \Omega_{\text {Der }}^{n, \alpha}\left(\mathbb{A}^{\bullet}\right), \forall n \in \mathbb{N}$.

i) The NC connection on $\mathbb{A}^{\bullet}$ is defined as a linear map of homogeneous degree $0 \nabla: \Omega_{\mathrm{Der}}^{0, \bullet} \rightarrow$ $\Omega_{\text {Der }}^{1, \bullet}$ such that

$$
\nabla(a)=\hat{d} a+A a, \quad \forall a \in \mathbb{A}^{\bullet}, \quad A \in \Omega_{\operatorname{Der}}^{1,0}\left(\mathbb{A}^{\bullet}\right),
$$

where $\hat{d}$ is the differential defined in ii) of Proposition 2.8. $A \in \Omega_{\mathrm{Der}}^{1,0}\left(\mathbb{A}^{\bullet}\right)$ is the 1 -form connection. 
ii) Let $h$ be a Hermitian structure on $\mathbb{A}^{\bullet}$. The NC connection on $\mathbb{A}^{\bullet}$ is Hermitian if it satisfies $X\left(h\left(a_{1}, a_{2}\right)\right)=h\left(\nabla_{X}\left(a_{1}\right), a_{2}\right)+(-1)^{|X|\left|a_{1}\right|} h\left(a_{1}, \nabla_{X}\left(a_{2}\right)\right), \forall a_{1} \in \mathbb{A} \bullet$ of homogenous degree $\left|a_{1}\right|, \forall a_{2} \in \mathbb{A}^{\bullet}$, and for any real derivation $X$ in $\operatorname{Der}\left(\mathbb{A}^{\bullet}\right)$ with homogeneous degree $|X|$.

iii) The curvature is defined as a linear map $F: \Omega_{\operatorname{Der}}^{0, \alpha}\left(\mathbb{A}^{\bullet}\right) \rightarrow \Omega_{\operatorname{Der}}^{2, \alpha}\left(\mathbb{A}^{\bullet}\right)$, such that

$$
F(a)=\nabla(\nabla(a))=(\hat{d} a+A A)(a), \quad \forall a \in \mathbb{A}^{\bullet} .
$$

Note that the connection defined here preserves the degree of the $\mathbb{Z}_{2}$-graduation,

$$
\nabla: \Omega_{\operatorname{Der}}^{0, \alpha}\left(\mathbb{A}^{\bullet}\right) \rightarrow \Omega_{\operatorname{Der}}^{1, \alpha}\left(\mathbb{A}^{\bullet}\right)
$$

Then, one has for any $X \in \operatorname{Der}^{\bullet}(\mathbb{A})$ of homogeneous degree $|X|,|A(X)|=\left|\nabla_{X}\right|=|X|$. Notice that we do not use a $\mathrm{NC}$ analogue of a graded connection. This latter appears to have interesting relationship with the concept of superconnection introduced by Quillen [45]. This will be reported elsewhere [46].

From Definition 2.7, one obtains the following relations that will be used in the sequel

Proposition 2.9. For any $X, Y \in \operatorname{Der}\left(\mathbb{A}^{\bullet}\right)$, any $z \in \mathcal{Z}\left(\mathbb{A}^{\bullet}\right)$ and any $a \in \mathbb{A}^{\bullet}$ of homogeneous degree $|X|,|Y|,|z|$ and $|a|$, one has

$$
\begin{aligned}
& \nabla_{X}(a)=X(a)+A(X) a ; \quad \nabla_{X}(a b)=\nabla_{X}(a) b+(-1)^{|X||a|} a X(b), \quad \forall b \in \mathbb{A}^{\bullet}, \\
& \nabla_{z X}(a)=z \nabla_{X}(a), \quad \nabla_{X z}(a)=(-1)^{|a||z|}\left(\nabla_{X}(a)\right) z, \\
& F(X, Y)(a)=\left(\left[\nabla_{X}, \nabla_{Y}\right]_{\bullet}-\nabla_{[X, Y] \bullet}\right)(a), \quad \forall a \in \mathbb{A}^{\bullet} .
\end{aligned}
$$

Let ${ }^{\dagger}$ denote the involution on $\mathbb{A}^{\bullet},(a b)^{\dagger}=(-1)^{|a||b|} b^{\dagger} a^{\dagger}$ and let $h_{0}\left(a_{1}, a_{2}\right)=a_{1}^{\dagger} a_{2}$ be the Hermitian structure on $\mathbb{A}^{\bullet}$. A NC connection on $\mathbb{A}^{\bullet}$ as given by Definition 2.7 is Hermitian when for any real derivation $X$ of homogeneous degree, the 1 -form connection $A \in \Omega_{\text {Der }}^{1,0}\left(\mathbb{A}^{\bullet}\right)$ satisfies $A(X)^{\dagger}=-A(X)$.

Proof. The relations (2.3)-(2.5) can be simply obtained from standard calculation. Then, assume that the Hermitian structure is given by $h_{0}\left(a_{1}, a_{2}\right)=a_{1}^{\dagger} a_{2}$ and consider a Hermitian NC connection $\nabla$. For any real derivation in $\operatorname{Der}\left(\mathbb{A}^{\bullet}\right)$ with homogeneous degree $|X|$ one has, on one hand, $X\left(h_{0}\left(a_{1}, a_{2}\right)\right)=X\left(a_{1}^{\dagger}\right) a_{2}+(-1)^{|X|\left|a_{1}\right|} a_{1} X\left(a_{2}\right)$ and on the other hand $h_{0}\left(\nabla_{X}\left(a_{1}\right), a_{2}\right)+$ $(-1)^{|X|\left|a_{1}\right|} h_{0}\left(a_{1}, \nabla_{X}\left(a_{2}\right)\right)=\left(X\left(a_{1}^{\dagger}\right)+(-1)^{|X|\left|a_{1}\right|} a_{1}^{\dagger} A^{\dagger}(X)\right) a_{2}+(-1)^{|X|\left|a_{1}\right|} a_{1}^{\dagger}\left(X\left(a_{2}\right)+A(X) a_{2}\right)$, using Proposition 2.9 and the reality of $X$. Then, the relation given in Definition 2.7

$$
X\left(h_{0}\left(a_{1}, a_{2}\right)\right) \mathcal{A}(X)=h_{0}\left(\nabla_{X}\left(a_{1}\right), a_{2}\right)+(-1)^{|X|\left|a_{1}\right|} h_{0}\left(a_{1}, \nabla_{X}\left(a_{2}\right)\right)
$$

is fulfilled provided $A^{\dagger}(X)=-A(X)$.

The gauge transformations are defined in a way which preserves the degree of the NC connection. In the following, we will focus on unitary gauge transformations that are compatible with the Hermitian structure.

Definition 2.8. The gauge group is defined as $\operatorname{Aut}^{0}\left(\mathbb{A}^{\bullet}\right)$, the group of the automorphisms with degree 0 of $\mathbb{A}^{\bullet}$. Let $a^{\varphi} \equiv \varphi(a) \forall \varphi \in \operatorname{Aut}^{0}\left(\mathbb{A}^{\bullet}\right), \forall a \in \mathbb{A}^{\bullet}$. Let $h$ be a Hermitian structure on $\mathbb{A}^{\bullet}$. The unitary gauge group is defined as the subgroup $\mathcal{U}\left(\mathbb{A}^{\bullet}\right) \subset \operatorname{Aut}^{0}\left(\mathbb{A}^{\bullet}\right)$ such that $\mathcal{U}\left(\mathbb{A}^{\bullet}\right)=\left\{\varphi \in \operatorname{Aut}^{0}\left(\mathbb{A}^{\bullet}\right) / h\left(a_{1}^{\varphi}, a_{2}^{\varphi}\right)=h\left(a_{1}, a_{2}\right), \forall a_{1}, a_{2} \in \mathbb{A}^{\bullet}\right\}$.

When $h\left(a_{1}, a_{2}\right)=h_{0}\left(a_{1}, a_{2}\right)=a_{1}^{\dagger} a_{2}$, it follows from this definition that the gauge group $\mathcal{U}\left(\mathbb{A}^{\bullet}\right)$ can be identified obviously with the group of unitary elements of $\mathbb{A}^{\bullet}$. 
Proposition 2.10. For any NC connection $\nabla$ as given in Definition 2.7 and for any $\varphi \in$ $\operatorname{Aut}^{0}\left(\mathbb{A}^{\bullet}\right)$, the linear map of homogeneous degree $0 \nabla^{\varphi}: \Omega_{\mathrm{Der}}^{0, \bullet} \rightarrow \Omega_{\mathrm{Der}}^{1, \bullet}$ such that $\nabla^{\varphi}(a)(X)=$ $\left(\varphi^{-1} \circ \nabla \circ \varphi\right)(a)(X), \forall a \in \mathbb{A}^{\bullet}, \forall X \in \operatorname{Der}\left(\mathbb{A}^{\bullet}\right)$ defines a $N C$ connection. The corresponding 1 -form connection is defined by $A^{\varphi}=\varphi^{-1}(\mathbb{I}) \hat{d} \varphi(\mathbb{I})+\varphi^{-1}(\mathbb{I}) A \varphi(\mathbb{I})$. The gauge transformation of the curvature is given by $F^{\varphi}(a)=\left(\varphi^{-1} \circ F \circ \varphi\right)(a), \forall a \in \mathbb{A}^{\bullet}$.

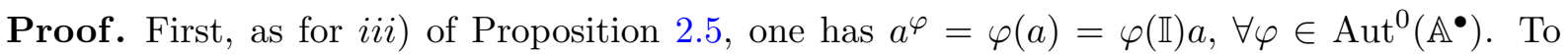
simplify the notations, we set $g=\varphi(\mathbb{I})$. Then, from Definition 2.7, one obtains $\varphi^{-1}(\nabla(\varphi(a)))=$ $g^{-1} \nabla(g a)=g^{-1} \hat{d}(g a)+g^{-1} A g a=\hat{d} a+\left(g^{-1} \hat{d} g+g^{-1} A g\right) a$. The expression for $A^{\varphi}$ follows. The gauge transformation for the curvature can be obtained by a simple calculation.

Proposition 2.6 and Lemma 2.1 must be slightly modified to take into account the total grading $\mathbb{N} \times \mathbb{Z}_{2}$. In fact, this can be compactly expressed in terms of differential forms. Note that the bracket on $\Omega_{\operatorname{Der}}\left(\mathbb{A}^{\bullet}\right)$ is $\left[\omega_{p}, \eta_{q}\right] \bullet=\omega \eta-(-1)^{p q}(-1)^{|\omega||\eta|} \eta \omega, \forall \omega \in \Omega_{\operatorname{Der}}^{p,|\omega|}\left(\mathbb{A}^{\bullet}\right), \forall \eta \in \Omega_{\operatorname{Der}}^{q,|\eta|}\left(\mathbb{A}^{\bullet}\right)$. The following lemma holds

Lemma 2.2. Assume that $\exists \eta \in \Omega_{\mathrm{Der}}^{1,0}\left(\mathbb{A}^{\bullet}\right) / \hat{d} a=[\eta, a] \bullet=\eta a-a \eta, \forall a \in \mathbb{A}^{\bullet}$ where $\hat{d}$ is the differential given in Proposition 2.8. Then, one has the properties:

i) The map $\nabla^{\text {inv }}: \Omega_{\text {Der }}^{0, \alpha}\left(\mathbb{A}^{\bullet}\right) \rightarrow \Omega_{\text {Der }}^{1, \alpha}\left(\mathbb{A}^{\bullet}\right), \alpha=0,1$ such that $\nabla^{\operatorname{inv}}(a)=\hat{d} a-\eta a=-a \eta$, $\forall a \in \mathbb{A}^{\bullet}$, defines a connection in the sense of Definition 2.7. It is called the canonical connection. $\nabla^{\text {inv }}$ is gauge invariant: $\left(\nabla^{\text {inv }}\right)^{g}(a)=\nabla^{\text {inv }}(a), \forall g \in \mathcal{U}\left(\mathbb{A}^{\bullet}\right)$.

ii) The curvature for the canonical connection is defined by the linear map $F^{\mathrm{inv}}: \Omega_{\mathrm{Der}}^{0, \alpha}\left(\mathbb{A}^{\bullet}\right) \rightarrow$ $\Omega_{\text {Der }}^{2, \alpha}\left(\mathbb{A}^{\bullet}\right)$ such that $F^{\text {inv }}(a)=(-\hat{d} \eta+\eta \eta)(a), \forall a \in \mathbb{A}^{\bullet}$.

iii) For any NC connection $\nabla$ in the sense of Definition 2.7, $\mathcal{A} \in \Omega_{\mathrm{Der}}^{1,0}\left(\mathbb{A}^{\bullet}\right)$ such that $\mathcal{A}(a)=$ $\left(\nabla-\nabla^{\mathrm{inv}}\right)(a)=(A+\eta)(a), \forall a \in \mathbb{A}^{\bullet}$ defines a tensor form. $\mathcal{A}(X), \forall X \in \operatorname{Der}\left(\mathbb{A}^{\bullet}\right)$ are called the covariant coordinates. The curvature for any NC connection $\nabla$ is defined by the linear map $F: \Omega_{\text {Der }}^{0, \alpha}\left(\mathbb{A}^{\bullet}\right) \rightarrow \Omega_{\text {Der }}^{2, \alpha}\left(\mathbb{A}^{\bullet}\right)$ such that $\left.F(a)=F^{\text {inv }}(a)+(\mathcal{A} \mathcal{A}+\hat{d} \mathcal{A}-[\mathcal{A}, \eta] \bullet)\right)(a)$, $\forall a \in \mathbb{A}^{\bullet}$.

iv) For any $X, Y \in \operatorname{Der}\left(\mathbb{A}^{\bullet}\right), \forall a \in \mathbb{A}^{\bullet}$ with respective homogeneous degree $|X|,|Y|$, $|a|$, the following relations hold:

$$
\begin{aligned}
& \nabla_{X}^{\operatorname{inv}}(a)=-(-1)^{|X||a|} a \eta(X), \quad \nabla_{X}(a)=\mathcal{A}(X) a-(-1)^{|X||a|} a \eta(X), \\
& F(X, Y)=[\mathcal{A}(X), \mathcal{A}(Y)] \bullet-\mathcal{A}\left([X, Y]_{\bullet}\right)+(\eta([X, Y] \bullet)-[\eta(X), \eta(Y)] \bullet), \\
& F^{\operatorname{inv}}(X, Y)=-[\eta(X), \eta(Y)] \bullet+\eta\left([X, Y]_{\bullet}\right) .
\end{aligned}
$$

Proof. From Definition 2.7, any 1-form in $\Omega_{\text {Der }}^{1,0}\left(\mathbb{A}^{\bullet}\right)$ defines a connection. In particular, pick $A=-\eta$. The property $i)$ follows. Then, $\forall g \in \mathcal{U}\left(\mathbb{A}^{\bullet}\right),\left(\nabla^{\text {inv }}\right)^{g}(a)=g^{\dagger} \nabla^{\text {inv }}(g a)=-g^{\dagger}(g a \eta)=$ $-a \eta=\nabla^{\operatorname{inv}}(a)$. The corresponding curvature is determined by the 2 -form $F=\hat{d} A+A A$ for $A=-\eta$. So, $F^{\text {inv }}=-\hat{d} \eta+\eta \eta$. The property for $\mathcal{A}$ in iii) is a direct consequence of the definition of a tensor form. The expression for $F$ in terms of $\mathcal{A}$ is obtained by setting $A=\mathcal{A}-\eta$ in Definition 2.7 and using the expression for $F^{\text {inv }}$. Finally, the relations given in $i v$ ) are obtained by using the main properties of the graded differential calculus given in Proposition 2.8. One has in particular $\mathcal{A} \mathcal{A}(X, Y)=[\mathcal{A}(X), \mathcal{A}(Y)]_{\bullet},[\mathcal{A}, \eta] \bullet(X, Y)=[\eta(X), \mathcal{A}(Y)]_{\bullet}-(-1)^{|X||Y|}[\eta(Y), \mathcal{A}(X)] \bullet$ $\hat{d} \mathcal{A}(X, Y)=[\eta(X), \mathcal{A}(Y)]_{\bullet}-(-1)^{|X||Y|}[\eta(Y), \mathcal{A}(X)]_{\bullet}-\mathcal{A}([X, Y] \bullet), \hat{d} \eta(X, Y)=2[\eta(X), \eta(Y)] \bullet-$ $\eta\left([X, Y]_{\bullet}\right)$. Then, the relations $(2.6)-(2.8)$ follow.

We will now apply the above non-graded and graded differential calculi based on the derivations of associative algebras to two cases. The first case deals with a non-graded differential calculus on the Moyal algebra $\mathcal{M}$ stemming from the maximal subalgebra of $\operatorname{Der}(\mathcal{M})$ whose 
elements can be interpreted as infinitesimal symplectomorphisms. This gives rise to a natural construction of Yang-Mills-Higgs type models defined on $\mathcal{M}$. The second case deals with a $\mathbb{Z}_{2^{-}}$ graded differential calculus on a $\mathbb{Z}_{2}$-graded associative algebra built from two copies of $\mathcal{M}$. Due to the grading, the gauge theory built from the square of the corresponding curvatures involves as contributions the action (1.2) derived in [26] and [27] as well the renormalisable NC $\varphi^{4}$-model with harmonic term elaborated in $[12,13]$.

From now on, the Hermitian structure is $h_{0}\left(a_{1}, a_{2}\right)=a_{1}^{\dagger} a_{2}$. According to the above discussion, the gauge group is the group of unitary elements of the associative algebra.

\section{Application: gauge theories on the Moyal algebra}

\subsection{General properties of the Moyal algebra}

In this subsection, we collect the properties of the Moyal algebra that will be used in the sequel. For more details, see e.g. $[7,8])$. Let $\mathcal{S}\left(\mathbb{R}^{D}\right) \equiv \mathcal{S}$ and $\mathcal{S}^{\prime}\left(\mathbb{R}^{D}\right) \equiv \mathcal{S}^{\prime}$, with $D=2 n$, be respectively the space of complex-valued Schwartz functions on $\mathbb{R}^{D}$ and the dual space of tempered distributions on $\mathbb{R}^{D}$. The complex conjugation in $\mathcal{S}, a \mapsto a^{\dagger}, \forall a \in \mathcal{S}$, defines a natural involution in $\mathcal{S}$ that can be extended to $\mathcal{S}^{\prime}$ by duality and that will be used in the rest of this paper. Let $\Theta_{\mu \nu}$ be an invertible constant skew-symmetric matrix which can be written as $\Theta=\theta \Sigma$ where $\Sigma$ is the "block-diagonal" matrix, $\Sigma=\operatorname{diag}(J, \ldots, J)$ involving $n(2 \times 2)$ matrix $J$ given by $J=\left(\begin{array}{cc}0 & -1 \\ 1 & 0\end{array}\right)$ and the parameter $\theta$ has mass dimension -2 . We use the notation $y \Theta^{-1} z \equiv y_{\mu} \Theta_{\mu \nu}^{-1} z^{\nu}$. The following proposition summarises properties relevant for the ensuing discussion:

Proposition 3.1. Let the $\star$-Moyal product be defined as $\star: \mathcal{S} \times \mathcal{S} \rightarrow \mathcal{S}$ by

$$
(a \star b)(x)=\frac{1}{(\pi \theta)^{D}} \int d^{D} y d^{D} z a(x+y) b(x+z) e^{-i 2 y \Theta^{-1} z}, \quad \forall a, b \in \mathcal{S} .
$$

Then, $(\mathcal{S}, \star)$ is a non unital associative involutive Fréchet algebra with faithful trace given by $\int d^{D} x(a \star b)(x)=\int d^{D} x(b \star a)(x)=\int d^{D} x a(x) \cdot b(x)$, where the symbol "” is the usual commutative product of functions in $\mathcal{S}$.

The $\star$ product $(3.1)$ can be further extended to $\mathcal{S}^{\prime} \times \mathcal{S} \rightarrow \mathcal{S}^{\prime}$ upon using duality of vector spaces: $\langle T \star a, b\rangle=\langle T, a \star b\rangle, \forall T \in \mathcal{S}^{\prime}, \forall a, b \in \mathcal{S}$. In a similar way, (3.1) can be extended to $\mathcal{S} \times \mathcal{S}^{\prime} \rightarrow \mathcal{S}^{\prime}$, via $\langle a \star T, b\rangle=\langle T, b \star a\rangle, \forall T \in \mathcal{S}^{\prime}, \forall a, b \in \mathcal{S}$. Then, the Moyal algebra is defined as $[7,8]$.

Definition 3.1. Let $\mathcal{M}_{L}$ and $\mathcal{M}_{R}$ be respectively defined by $\mathcal{M}_{L}=\left\{T \in \mathcal{S}^{\prime} / a \star T \in \mathcal{S}, \forall a \in \mathcal{S}\right\}$ and $\mathcal{M}_{R}=\left\{T \in \mathcal{S}^{\prime} / T \star a \in \mathcal{S}, \forall a \in \mathcal{S}\right\}$. Then, the Moyal algebra $\mathcal{M}$ is defined by

$$
\mathcal{M}=\mathcal{M}_{L} \cap \mathcal{M}_{R}
$$

Notice that $\mathcal{M}_{L}$ and $\mathcal{M}_{R}$ are sometimes called in the literature respectively the left and right multiplier algebras. By construction, $\mathcal{S}$ is a two-sided ideal of $\mathcal{M}$. The essential structural properties of the Moyal algebra that we will need are summarised in the following proposition:

Proposition 3.2. $\mathcal{M}, \star$ is a maximal unitisation of $(\mathcal{S}, \star)$. It is a locally convex associative unital *-algebra. It involves the plane waves, the Dirac distribution and its derivatives and the polynomial functions. 
In the following, we will need the following asymptotic formula for the $\star$-product

$$
\begin{aligned}
(a \star b)(x)= & a(x) \cdot b(x) \\
& +\left.\sum_{n=1}^{\infty} \frac{1}{n !}\left(\frac{i}{2} \Theta^{\mu_{1} \nu_{1}} \frac{\partial}{\partial x^{\mu_{1}}} \frac{\partial}{\partial y^{\nu_{1}}}\right) \ldots\left(\frac{i}{2} \Theta^{\mu_{n} \nu_{n}} \frac{\partial}{\partial x^{\mu_{n}}} \frac{\partial}{\partial y^{\nu_{n}}}\right) a(x) b(y)\right|_{x=y},
\end{aligned}
$$

which holds for any polynomial functions $a, b$. For a detailed study of the validity of (3.2), see e.g. [47].

Proposition 3.3. The center is $\mathcal{Z}(\mathcal{M})=\mathbb{C}$.

Other relevant properties of the $\star$-product that hold on $\mathcal{M}$ and will be used in the sequel are

Proposition 3.4. For any $a, b \in \mathcal{M}$, one has the following relations on $\mathcal{M}$ :

$$
\begin{aligned}
& \partial_{\mu}(a \star b)=\partial_{\mu} a \star b+a \star \partial_{\mu} b, \quad(a \star b)^{\dagger}=b^{\dagger} \star a^{\dagger}, \quad\left[x_{\mu}, a\right]_{\star}=i \Theta_{\mu \nu} \partial_{\nu} a \\
& x_{\mu} \star a=\left(x_{\mu} \cdot a\right)+\frac{i}{2} \Theta_{\mu \nu} \partial_{\nu} a, \quad x_{\mu}(a \star b)=\left(x_{\mu} a\right) \star b-\frac{i}{2} \Theta_{\mu \nu} a \star \partial_{\nu} b \\
& \left(x_{\mu} \cdot x_{\nu}\right) \star a=x_{\mu} \cdot x_{\nu} \cdot a+\frac{i}{2}\left(x_{\mu} \Theta_{\nu \beta}+x_{\nu} \Theta_{\mu \beta}\right) \partial_{\beta} a-\frac{1}{4} \Theta_{\mu \alpha} \Theta_{\nu \sigma} \partial_{\alpha} \partial_{\sigma} a \\
& a \star\left(x_{\mu} \cdot x_{\nu}\right)=x_{\mu} \cdot x_{\nu} \cdot a-\frac{i}{2}\left(x_{\mu} \Theta_{\nu \beta}+x_{\nu} \Theta_{\mu \beta}\right) \partial_{\beta} a-\frac{1}{4} \Theta_{\mu \alpha} \Theta_{\nu \sigma} \partial_{\alpha} \partial_{\sigma} a \\
& \left.\left[\left(x_{\mu} \cdot x_{\nu} \cdot x_{\rho}\right), a\right]_{\star}=i\left(x_{\rho} x_{\mu} \Theta_{\nu \beta}+x_{\nu} x_{\rho} \Theta_{\mu \beta}+x_{\mu} x_{\nu} \Theta_{\rho \beta}\right) \partial_{\beta} a-\frac{i}{4} \Theta_{\mu \alpha} \Theta_{\nu \sigma} \Theta_{\rho \lambda} \partial_{\alpha} \partial_{\sigma} \partial_{\lambda} a\right)
\end{aligned}
$$

Proof. The relations can be obtained by calculations, using from instance the asymptotic expansion (3.2).

Notice that, as a special case of the last relation (3.3a), one obtains the celebrated relation among the "coordinate functions" defined on $\mathcal{M}$ :

$$
\left[x_{\mu}, x_{\nu}\right]_{\star}=i \Theta_{\mu \nu}
$$

where we set $[a, b]_{\star} \equiv a \star b-b \star a$.

As a final remark, note that the Moyal algebra has $\mathcal{Z}(\mathcal{M})=\mathbb{C}$ as trivial center, which stems from the fact that $\Theta_{\mu \nu}$ is non degenerate. This simplifies the situation regarding all the structures of modules over $\mathcal{Z}(\mathcal{M})$ that are involved in the present algebraic scheme. In the present case, these are simply replaced by vector spaces over $\mathbb{C}$.

\subsection{Differential calculus and inner derivations}

The vector space of derivations of $\mathcal{M}$ is infinite dimensional. Then, a differential calculus based on the full derivation algebra $\operatorname{Der}(\mathcal{M})$ would give rise to gauge potentials with an infinite number of components. In view of the construction of physically oriented gauge theories on Moyal spaces, it is more convenient to deal with gauge potentials having a finite number of components. These occur within restricted differential calculi based on Lie subalgebras of $\operatorname{Der}(\mathbb{A})$, as given in Proposition 2.3. In the following, we will consider two Lie subalgebras of $\operatorname{Der}(\mathcal{M})$, denoted by $\mathcal{G}_{1}$ and $\mathcal{G}_{2}$. The first one is Abelian and is simply related to the "spatial derivatives" $\partial_{\mu}$. The resulting differential calculus underlies almost all the constructions of NCFT defined on Moyal spaces. For further convenience, we set from now on

$$
\partial_{\mu} a=\left[i \xi_{\mu}, a\right]_{\star}, \quad \xi_{\mu}=-\Theta_{\mu \nu}^{-1} x^{\nu}, \quad \forall a \in \mathcal{M} .
$$

The second derivation Lie subalgebra $\mathcal{G}_{2}$, such that $\mathcal{G}_{1} \subset \mathcal{G}_{2}$, is the maximal subalgebra of $\operatorname{Der}(\mathcal{M})$ whose derivations can be interpreted as infinitesimal symplectomorphisms. Notice that in each case, the existence of $\mathrm{NC}$ connections is explicitly verified, due to the existence of canonical gauge invariant connections of the type given in Proposition 2.6. 
Proposition 3.5. Let $\mathcal{P}_{2} \subset \mathcal{M}$ denote the set of polynomial functions with degree $d \leq 2$. Let $\{a, b\}_{P B} \equiv \Theta_{\mu \nu} \frac{\partial a}{\partial x_{\mu}} \frac{\partial b}{\partial x_{\nu}}$ for any polynomial function $a, b \in \mathcal{M}$ denote the Poisson bracket for the symplectic structure defined by $\Theta_{\mu \nu}$. Then, $\mathcal{P}_{2}$ equipped with the Moyal bracket [, $]_{\star}$ is a Lie algebra which satisfies

$$
\left[P_{1}, P_{2}\right]_{\star}=i\left\{P_{1}, P_{2}\right\}_{P B}, \quad \forall P_{1}, P_{2} \in \mathcal{P}_{2} .
$$

Proof. Using (3.2), one infers that $\left(P_{1} \star P_{2}\right)(x), \forall P_{1}, P_{2} \in \mathcal{P}_{2}$ truncate to a finite expansion. Namely, $\left(P_{1} \star P_{2}\right)(x)=P_{1}(x) \cdot P_{2}(x)+\frac{i}{2} \Theta_{\mu \nu} \frac{\partial P_{1}}{\partial x_{\mu}} \frac{\partial P_{2}}{\partial x_{\nu}}-\frac{1}{4} \Theta_{\mu \nu} \Theta_{\rho \sigma} \frac{\partial^{2} P_{1}}{\partial x_{\mu} \partial x_{\rho}} \frac{\partial P_{2}}{\partial x_{\nu} \partial x_{\sigma}}$ where the last term is a constant. Then, $\left[P_{1}, P_{2}\right]_{\star}=i \Theta_{\mu \nu} \frac{\partial P_{1}}{\partial x_{\mu}} \frac{\partial P_{2}}{\partial x_{\nu}}$ from which follows the proposition.

Consider now the Lie subalgebra $\mathcal{G}_{2} \subset \operatorname{Der}(\mathcal{M})$ which is the image of $\mathcal{P}_{2}$ by $\operatorname{Ad}, \mathcal{G}_{2}=\{X \in$ $\operatorname{Der}\left(\mathcal{M} / X=\operatorname{Ad}_{P}, P \in \mathcal{P}_{2}\right\}$. In order to apply Proposition 2.6 and Lemma 2.1 to the present situation, one has to define properly the 1-form $\eta$ from which most of the objects entering the construction of gauge theories are derived. To do this, one defines the linear map $\eta$ as

$$
\eta: \quad \mathcal{G}_{2} \rightarrow \mathcal{P}_{2} \quad / \quad \eta(X)=P-P(0), \quad \forall X \in \mathcal{G}_{2},
$$

where $P(0) \in \mathbb{C}$ is the evaluation of the polynomial function $P$ at $x=0$. Then, $X(a)=\operatorname{Ad}_{P}(a)=$ $\operatorname{Ad}_{\eta(X)}(a), \forall X \in \mathcal{G}_{2}, \forall a \in \mathcal{M}$ and (3.6) can be used to define the 1-form $\eta$ in the present case. Notice that $\eta$ does not define a morphism of Lie algebra since, as implied by the property $i$ ) of Lemma 2.1, one has $\eta\left(\left[X_{1}, X_{2}\right]\right)-\left[\eta\left(X_{1}\right), \eta\left(X_{2}\right)\right] \in \mathbb{C}$ and is non zero in the present case. Indeed, take for instance $\eta\left(\partial_{\mu}\right)=i \xi_{\mu}($ see $(3.5))$; then $\eta\left(\left[\partial_{\mu}, \partial_{\nu}\right]\right)-\left[\eta\left(\partial_{\mu}\right), \eta\left(\partial_{\nu}\right)\right]_{\star}=\left[\xi_{\mu}, \xi_{\nu}\right]_{\star}=-i \Theta_{\mu \nu}^{-1}$.

At this point, some remarks are in order. Proposition 3.5 singles out two subalgebras of derivations, whose elements are related to infinitesimal symplectomorphisms. These are sometimes called area-preserving diffeomorphims in the physics literature. The first algebra $\mathcal{G}_{1}$ is Abelian and is simply the image by Ad of the algebra generated by $\left\{P_{\mu}=x_{\mu}, \mu=1, \ldots, D\right\}$, i.e. the polynomials with degree 1 . It is the algebra related to the spatial derivatives $\partial_{\mu}$ in view of the 3rd relation of (3.3a) and (3.5). One has immediately, due to (3.4)

$$
\left[\partial_{\mu}, \partial_{\nu}\right](a)=\left[\operatorname{Ad}_{i \xi_{\mu}}, \operatorname{Ad}_{i \xi_{\nu}}\right](a)=\operatorname{Ad}_{\left[i \xi_{\mu}, i \xi_{\nu}\right]_{\star}}(a)=0, \quad \forall a \in \mathcal{M} .
$$

Note that the interpretation of $\left[x_{\mu}, a\right]_{\star}$ as the Lie derivative along the (constant) Hamiltonian vector field $i \Theta_{\mu \nu}$ is obvious. The differential calculus based on $\mathcal{G}_{1}$ is the minimal one that can be set-up on the Moyal algebra and actually underlies most of the studies of the NCFT on Moyal spaces. The second algebra $\mathcal{G}_{2}$ is the image by Ad of $\left\{P_{\mu \nu}=\left(x_{\mu} x_{\nu}\right), \mu, \nu=1, \ldots, D\right\}$, the algebra generated by the polynomials with degree 2 . It is the maximal subalgebra of $\operatorname{Der}(\mathcal{M})$ whose elements can be related to symplectomorphims. Observe that from (3.3c) and (3.3d) one has

$$
\left[\left(x_{\mu} \cdot x_{\nu}\right), a\right]_{\star}=i\left(x_{\mu} \Theta_{\nu \beta}+x_{\nu} \Theta_{\mu \beta}\right) \partial_{\beta} a,
$$

so that the bracket in the LHS can again be interpreted as the Lie derivative along a Hamiltonian vector field. Note that this is no longer true for polynomials with degree $d \geq 3$, which is apparent from (3.3e) for $d=3$.

Once $\mathcal{G}_{1}$ or $\mathcal{G}_{2}$ is choose and the corresponding 1-form $\eta$ is determined, all the properties and mathematical status of the various objects entering the construction of gauge theories on Moyal spaces are entirely fixed from Proposition 2.6 and Lemma 2.1. The corresponding relations are summarised below for further convenience. For any $X \in \mathcal{G}_{i}, i=1,2$, one has

$$
\begin{aligned}
& \nabla_{X}^{\text {inv }}(a)=-a \star \eta(X), \quad \mathcal{A}(X)=A(X)+\eta(X), \\
& \nabla_{X}(a)=\nabla_{X}^{\operatorname{inv}}(a)+\mathcal{A}_{X} \star a=\nabla_{X}^{\operatorname{inv}}(a)+(A(X)+\eta(X)) \star a=X(a)+A(X) \star a, \\
& F(X, Y)=([\mathcal{A}(X), \mathcal{A}(Y)]-\mathcal{A}[X, Y])-([\eta(X), \eta(Y)]-\eta[X, Y]) .
\end{aligned}
$$




\subsection{Application to the Moyal space}

Consider first the Abelian algebra $\mathcal{G}_{1}$ generated by the spatial derivatives (3.5). Then, after doing a simple rescaling $A(X) \rightarrow-i A(X)$ (i.e. defining $\nabla_{X}(\mathbb{I}) \equiv-i A(X)$ so that Hermitian connections satisfy $A(X)^{\dagger}=A(X)$ for any real derivation $\left.X\right)$ in order to make contact with the notations of e.g. $[26,15]$, and defining $\eta\left(\partial_{\mu}\right) \equiv \eta_{\mu}, \mathcal{A}\left(\partial_{\mu}\right) \equiv \mathcal{A}_{\mu}, A\left(\partial_{\mu}\right) \equiv A_{\mu}, F\left(\partial_{\mu}, \partial_{\nu}\right) \equiv F_{\mu \nu}$, $\mu=1, \ldots, D$, a straightforward application of (3.6), and (3.7)-(3.9) yields

$$
\begin{aligned}
& \eta_{\mu}=i \xi_{\mu}, \quad \nabla_{\mu}^{\operatorname{inv}}(a)=-i a \star \xi_{\mu}, \quad \forall a \in \mathcal{M}, \\
& \mathcal{A}_{\mu}=-i\left(A_{\mu}-\xi_{\mu}\right), \quad \nabla_{\mu}(a)=-i a \star \xi_{\mu}+\mathcal{A}_{\mu} \star a=\partial_{\mu} a-i A_{\mu} \star a, \quad \forall a \in \mathcal{M}, \\
& F_{\mu \nu}=\left[\mathcal{A}_{\mu}, \mathcal{A}_{\nu}\right]_{\star}-i \Theta_{\mu \nu}^{-1}=-i\left(\partial_{\mu} A_{\nu}-\partial_{\nu} A_{\mu}-i\left[A_{\mu}, A_{\nu}\right]_{\star}\right),
\end{aligned}
$$

which fix the respective mathematical status of the objects involved in most of the studies of NCFT on Moyal spaces. The group of (unitary) gauge transformation is the group of unitary elements of $\mathcal{M}, \mathcal{U}(\mathcal{M})$, as defined in Section 2 and one has

$$
\begin{aligned}
& A_{\mu}^{g}=g \star A_{\mu} \star g^{\dagger}+i g \star \partial_{\mu} g^{\dagger}, \quad \mathcal{A}_{\mu}^{g}=g \star \mathcal{A}_{\mu} \star g^{\dagger}, \\
& F_{\mu \nu}^{g}=g \star F_{\mu \nu} \star g^{\dagger}, \quad \forall g \in \mathcal{U}(\mathcal{M}) .
\end{aligned}
$$

Consider now the algebra $\mathcal{G}_{2}$. Let $\overline{\mathcal{G}}_{2} \subset \mathcal{G}_{2}$ denote the subalgebra of $\mathcal{G}_{2}$ whose image in $\mathcal{M}$ by the map $\eta$ (3.6) corresponds to the monomials of degree 2 . The image involves $\frac{D(D+1)}{2}$ elements defined by

$$
\eta\left(X_{\mu \nu}\right)=i \xi_{\mu} \xi_{\nu} \equiv \eta_{(\mu \nu)}, \quad \forall X_{\mu \nu} \in \overline{\mathcal{G}_{2}}, \quad \forall \mu, \nu=1, \ldots, D .
$$

The symbol $(\mu \nu)$ denotes symmetry under the exchange $\mu \leftrightarrow \nu$. Notice that the definition for $\eta_{\mu \nu}$ corresponds to real derivations. One has

$$
\left[\eta_{(\mu \nu)}, \eta_{(\rho \sigma)}\right]_{\star}=-\left(\Theta_{\rho \nu}^{-1} \eta_{(\mu \sigma)}+\Theta_{\sigma \nu}^{-1} \eta_{(\mu \rho)}+\Theta_{\rho \mu}^{-1} \eta_{(\nu \sigma)}+\Theta_{\sigma \mu}^{-1} \eta_{(\nu \rho)}\right) .
$$

which define the generic commutation relations for the $s p(2 n, \mathbb{R})$ algebra. Then, the algebra $\mathcal{G}_{2}$ we choose is generated by $\left\{\partial_{\mu}, X_{\mu \nu}\right\}$. Its image in $\mathcal{M}$ by the map $\eta(3.6)$ is the algebra $i s p(2 n, \mathbb{R})$. One has the additional commutation relations

$$
\left[\eta_{\mu}, \eta_{(\rho \sigma)}\right]_{\star}=\left(\Theta_{\mu \rho}^{-1} \eta_{\sigma}+\Theta_{\mu \sigma}^{-1} \eta_{\rho}\right) .
$$

Notice that any derivation related to $i s p(2 n, \mathbb{R})$ can be viewed as generating an infinitesimal symplectomorphism, as discussed above. Accordingly, the subalgebra $\mathcal{G}_{1} \subset \mathcal{G}_{2}$ can actually be interpreted physically as corresponding to spatial directions while $\overline{\mathcal{G}}_{2}$ corresponds to (symplectic) rotations. Notice also that in the case $D=2$, upon defining

$$
\eta_{X_{1}}=\frac{i}{4 \sqrt{2} \theta}\left(x_{1}^{2}+x_{2}^{2}\right), \quad \eta_{X 2}=\frac{i}{4 \sqrt{2} \theta}\left(x_{1}^{2}-x_{2}^{2}\right), \quad \eta_{X 3}=\frac{i}{2 \sqrt{2} \theta}\left(x_{1} x_{2}\right)
$$

one would obtain the following commutation relations

$$
\begin{array}{ll}
{\left[\eta_{X 1}, \eta_{X 2}\right]_{\star}=\frac{1}{\sqrt{2}} \eta_{X 3},} & {\left[\eta_{X 2}, \eta_{X 3}\right]_{\star}=-\frac{1}{\sqrt{2}} \eta_{X 1}, \quad\left[\eta_{X 3}, \eta_{X 1}\right]_{\star}=\frac{1}{\sqrt{2}} \eta_{X 2},} \\
{\left[\eta_{1}, \eta_{X 1}\right]_{\star}=\frac{1}{2 \sqrt{2}} \eta_{2},} & {\left[\eta_{2}, \eta_{X 1}\right]_{\star}=-\frac{1}{2 \sqrt{2}} \eta_{1},} \\
{\left[\eta_{1}, \eta_{X 2}\right]_{\star}=\frac{1}{2 \sqrt{2}} \eta_{2},} & {\left[\eta_{2}, \eta_{X 2}\right]_{\star}=\frac{1}{2 \sqrt{2}} \eta_{1},} \\
{\left[\eta_{1}, \eta_{X 3}\right]_{\star}=-\frac{1}{2 \sqrt{2}} \eta_{1},} & {\left[\eta_{2}, \eta_{X 3}\right]_{\star}=\frac{1}{2 \sqrt{2}} \eta_{2},}
\end{array}
$$


therefore making contact with the work carried out in [42]. Note that [42] did not consider the construction of gauge theories on Moyal spaces but was only focused on the construction of subalgebras of the $D=4$ Moyal algebra starting from a set of constraints forming a (subalgebra of a) $s p(2 n, \mathbb{R})$ algebra and the obtention of the algebra of smooth functions of $\mathbb{R}^{3}$ from a commutative limit.

A direct application of (3.6), and (3.7)-(3.9) permits one to determine the invariant connection and the tensor form. One obtains

$$
\begin{aligned}
& \nabla^{\mathrm{inv}}\left(\partial_{\mu}\right)(a) \equiv \nabla_{\mu}^{\mathrm{inv}}(a)=-i a \star \xi_{\mu}, \quad \nabla^{\mathrm{inv}}\left(X_{\mu \nu}\right)(a) \equiv \nabla_{(\mu \nu)}^{\mathrm{inv}}(a)=-i a \star\left(\xi_{\mu} \xi_{\nu}\right), \\
& \mathcal{A}\left(\partial_{\mu}\right) \equiv \mathcal{A}_{\mu}=-i\left(A_{\mu}-\xi_{\mu}\right), \quad \mathcal{A}\left(X_{(\mu \nu)}\right) \equiv \mathcal{A}_{(\mu \nu)}=-i\left(A_{(\mu \nu)}-\xi_{\mu} \xi_{\nu}\right),
\end{aligned}
$$

where the subscript $(\mu \nu)$ denotes symmetry under the exchange of $\mu$ and $\nu$. Then, any NC connection is obtained as the sum of the canonical connection and the tensor form, namely

$$
\begin{aligned}
& \nabla_{\mu}(a)=\nabla_{\mu}^{\operatorname{inv}}(a)+\mathcal{A}_{\mu} \star a=\partial_{\mu} a-i A_{\mu} \star a, \\
& \nabla_{(\mu \nu)}(a)=\nabla_{(\mu \nu)}^{\operatorname{inv}}(a)+\mathcal{A}_{(\mu \nu)} \star a=\left[i \xi_{\mu} \xi_{\nu}, a\right]_{\star}-i A_{(\mu \nu)} \star a .
\end{aligned}
$$

From this, one obtains the following expressions for the curvature

Proposition 3.6. Consider the differential calculus based on the maximal subalgebra of derivations of the Moyal algebra related to symplectomorphisms. The components of the 2-form curvature of a NC connection defined by a tensor 1-form with components $\mathcal{A}_{\mu}, \mathcal{A}_{(\mu \nu)}$ are given by

$$
\begin{aligned}
& F\left(\partial_{\mu}, \partial_{\nu}\right) \equiv F_{\mu \nu}=\left[\mathcal{A}_{\mu}, \mathcal{A}_{\nu}\right]_{\star}-i \Theta_{\mu \nu}^{-1}, \\
& F\left(\partial_{\mu}, X_{(\rho \sigma)}\right) \equiv F_{\mu(\rho \sigma)}=\left[\mathcal{A}_{\mu}, \mathcal{A}_{(\rho \sigma)}\right]_{\star}-\Theta_{\mu \rho}^{-1} \mathcal{A}_{\sigma}-\Theta_{\mu \sigma}^{-1} \mathcal{A}_{\rho}, \\
& \quad F\left(X_{(\mu \nu)}, X_{(\rho \sigma)}\right) \equiv F_{(\mu \nu)(\rho \sigma)}=\left[\mathcal{A}_{(\mu \nu)}, \mathcal{A}_{(\rho \sigma)}\right]_{\star}+\Theta_{\rho \nu}^{-1} \mathcal{A}_{(\mu \sigma)} \\
& \quad+\Theta_{\sigma \nu}^{-1} \mathcal{A}_{(\mu \rho)}+\Theta_{\rho \mu}^{-1} \mathcal{A}_{(\nu \sigma)}+\Theta_{\sigma \mu}^{-1} \mathcal{A}_{(\nu \rho)} .
\end{aligned}
$$

Proof. Use $F^{\text {inv }}(X, Y) \equiv \eta[X, Y]-[\eta(X), \eta(Y)]$ to evaluate the curvature for the canonical connection. Consider first $F_{\mu \nu}^{\text {inv }}$. From linearity of $\eta,\left[\partial_{\mu}, \partial_{\nu}\right]=0$ and $\left[\eta_{\mu}, \eta_{\nu}\right]=i \Theta_{\mu \nu}^{-1}$, one finds $F_{\mu \nu}^{\mathrm{inv}}=-i \Theta_{\mu \nu}^{-1}$. Then, from (3.9), one gets (3.13). To obtain (3.14), compute $\left[\partial_{\mu}, X_{(\rho \sigma)}\right](a)=$ $\left[\operatorname{Ad}_{\eta_{\mu}}, \operatorname{Ad}_{\eta_{(\rho \sigma)}}\right]=\operatorname{Ad}_{\left[\eta_{\mu}, \eta_{\rho \sigma}\right]_{\star}}$ using (3.11). This yields $\left[\partial_{\mu}, X_{(\rho \sigma)}\right](a)=\Theta_{\mu \rho}^{-1} \partial_{\sigma} a+\Theta_{\mu \sigma}^{-1} \partial_{\rho} a$ so that $\eta\left(\left[\partial_{\mu}, X_{(\rho \sigma)}\right]\right)=\Theta_{\mu \rho}^{-1} \eta_{\sigma}+\Theta_{\mu \sigma}^{-1} \eta_{\rho}$ which yields $F_{\mu(\rho \sigma)}^{\text {inv }}=0$. This combined with (3.9) yields (3.14). For (3.15), compute $\left[X_{(\mu \nu)}, X_{(\rho \sigma)}\right](a)=\operatorname{Ad}_{\left[\eta_{(\mu \nu)}, \eta_{(\rho \sigma)}\right]_{\star}}$ using (3.10). A straightforward calculation yields $F_{(\mu \nu)(\rho \sigma)}^{\text {inv }}=0$. From this follows $(3.15)$.

The gauge transformations are

$$
\begin{aligned}
& A_{\mu}^{g}=g^{\dagger} \star A_{\mu} \star g+i g^{\dagger} \star \partial_{\mu} g, \\
& A_{(\mu \nu)}^{g}=g^{\dagger} \star A_{(\mu \nu)} \star g+i g^{\dagger} \star\left(\xi_{\mu} \partial_{\nu}+\xi_{\nu} \partial_{\mu}\right) g, \quad \forall g \in \mathcal{U}(\mathcal{M}), \\
& \mathcal{A}_{\mu}^{g}=g^{\dagger} \star \mathcal{A}_{\mu} \star g, \quad \mathcal{A}_{(\mu \nu)}^{g}=g^{\dagger} \star \mathcal{A}_{(\mu \nu)} \star g, \quad \forall g \in \mathcal{U}(\mathcal{M}), \\
& F_{\mu \nu}^{g}=g^{\dagger} \star F_{\mu \nu} \star g, \quad F_{(\mu \nu)(\rho \sigma)}^{g}=g^{\dagger} \star F_{(\mu \nu)(\rho \sigma)} \star g, \quad \forall g \in \mathcal{U}(\mathcal{M}) \text {. }
\end{aligned}
$$

A possible construction of a NC gauge theory defined from the curvature (3.13)-(3.15) can be done as follows. Let $[x]$ denote the mass dimension ${ }^{2}$ of the quantity $x$. First, perform the rescaling $\eta_{(\mu \nu)} \rightarrow \mu \theta \eta_{(\mu \nu)}$ where $\mu$ is a parameter with $[\mu]=1$ that will fix the mass scale of the

\footnotetext{
${ }^{2}$ We work in the units $\hbar=c=1$.
} 
Higgs field to be identified in a while. Accordingly, the commutation relations (3.10), (3.11) are modified as

$$
\begin{aligned}
& {\left[\eta_{(\mu \nu)}, \eta_{(\rho \sigma)}\right]_{\star}=-\mu \theta\left(\Theta_{\rho \nu}^{-1} \eta_{(\mu \sigma)}+\Theta_{\sigma \nu}^{-1} \eta_{(\mu \rho)}+\Theta_{\rho \mu}^{-1} \eta_{(\nu \sigma)}+\Theta_{\sigma \mu}^{-1} \eta_{(\nu \rho)}\right),} \\
& {\left[\eta_{\mu}, \eta_{(\rho \sigma)}\right]_{\star}=\mu \theta\left(\Theta_{\mu \rho}^{-1} \eta_{\sigma}+\Theta_{\mu \sigma}^{-1} \eta_{\rho}\right),}
\end{aligned}
$$

and the components of the curvature become

$$
\begin{aligned}
& F_{\mu(\rho \sigma)}=\left[\mathcal{A}_{\mu}, \mathcal{A}_{(\rho \sigma)}\right]_{\star}-\mu \theta\left(\Theta_{\mu \rho}^{-1} \mathcal{A}_{\sigma}-\Theta_{\mu \sigma}^{-1} \mathcal{A}_{\rho}\right), \\
& F_{(\mu \nu)(\rho \sigma)}=\left[\mathcal{A}_{(\mu \nu)}, \mathcal{A}_{(\rho \sigma)}\right]_{\star}+\mu \theta\left(\Theta_{\rho \nu}^{-1} \mathcal{A}_{(\mu \sigma)}+\Theta_{\sigma \nu}^{-1} \mathcal{A}_{(\mu \rho)}+\Theta_{\rho \mu}^{-1} \mathcal{A}_{(\nu \sigma)}+\Theta_{\sigma \mu}^{-1} \mathcal{A}_{(\nu \rho)}\right)
\end{aligned}
$$

with (3.13) unchanged. Next, introduce a dimensionfull coupling constant $\alpha$ with mass dimension $[\alpha]=2-n(D=2 n)$. The functional $\mathcal{U}(\mathcal{M})$-gauge invariant action is then defined by

$$
S\left(A_{\mu}, \mathcal{A}_{(\mu \nu)}\right)=-\frac{1}{\alpha^{2}} \int d^{2 n} x\left(F_{\mu \nu} \star F_{\mu \nu}+F_{\mu(\rho \sigma)} \star F_{\mu(\rho \sigma)}+F_{(\mu \nu)(\rho \sigma)} \star F_{(\mu \nu)(\rho \sigma)}\right)
$$

and is chosen to depend on the fields $A_{\mu}$ and $\mathcal{A}_{\mu \nu}$. The mass dimensions are $\left[A_{\mu}\right]=\left[\mathcal{A}_{\mu}\right]=$ $\left[\mathcal{A}_{(\mu \nu)}\right]=1$.

Several remarks are now in order. First, the purely spatial part (3.13) takes the expected form when expressed in term of $A_{\mu}$ through the 1st relation (3.12), namely one obtains easily $F_{\mu \nu}=-i\left(\partial_{\mu} A_{\nu}-\partial_{\nu} A_{\mu}-i\left[A_{\mu}, A_{\nu}\right]_{\star}\right)$.

Then, one observes that $F_{\mu(\rho \sigma)}$ can be reexpressed as

$$
F_{\mu(\rho \sigma)}=D_{\mu}^{A} \mathcal{A}_{(\rho \sigma)}-\mu \theta\left(\Theta_{\mu \rho}^{-1} \mathcal{A}_{\sigma}-\Theta_{\mu \sigma}^{-1} \mathcal{A}_{\rho}\right), \quad D_{\mu}^{A} \mathcal{A}_{(\rho \sigma)} \equiv \partial_{\mu} \mathcal{A}_{(\rho \sigma)}-i\left[A_{\mu}, \mathcal{A}_{(\rho \sigma)}\right]_{\star}
$$

using (3.5) and (3.12). $D_{\mu}^{A} \mathcal{A}_{(\rho \sigma)}$ can be interpreted as a "covariant derivative" describing a NC analogue of the minimal coupling to the covariant field $\mathcal{A}_{\mu \nu}$. Besides, one has

$$
\begin{aligned}
-\frac{1}{\alpha} \int d^{2 n} x F_{\mu(\rho \sigma)} \star F_{\mu(\rho \sigma)}= & -\frac{1}{\alpha} \int d^{2 n} x\left(D_{\mu}^{A} \mathcal{A}_{(\rho \sigma)}\right)^{2} \\
& -4 \mu \theta\left(D_{\mu}^{A} \mathcal{A}_{(\rho \sigma)}\right) \Theta_{\mu \sigma}^{-1} \mathcal{A}_{\sigma}+(4 n+2) \mu^{2} \mathcal{A}_{\mu} \mathcal{A}_{\mu} .
\end{aligned}
$$

In view of the last term in (3.17), the gauge invariant action (3.16) involves a mass term for the gauge potential proportional to $\sim \frac{(4 n+2) \mu^{2}}{\alpha} A_{\mu} A_{\mu}$. Therefore, bare mass terms for $A_{\mu}$ can appear while preserving the gauge invariant of the action. Notice that the translational invariance of the action is broken by the term $(4 n+2) \mu^{2} \mathcal{A}_{\mu} \mathcal{A}_{\mu}$ in view of $\mathcal{A}_{\mu}=-i\left(A_{\mu}-\xi_{\mu}\right)$. Translational invariance is obviously maintened whenever in the action the gauge covariant curvature $F_{\mu(\rho \sigma)}$ is replaced by the gauge covariant derivative $D_{\mu}^{A} \mathcal{A}_{(\rho \sigma)}$.

The fact that $D_{\mu}^{A} \mathcal{A}_{(\rho \sigma)}$ in (3.16) can be viewed as a NC analogue of the covariant derivative of $\frac{D(D+1)}{2}$ scalar fields $\mathcal{A}_{\mu \nu}$, is very reminiscent of a Yang-Mills-Higgs action for which the covariant coordinates $\mathcal{A}_{\mu \nu}$ play the role of Higgs fields. Then, the last term in the action (3.16) which is the square of $F_{(\mu \nu)(\rho \sigma)}$ can be interpreted as the Higgs (quartic) potential part. Therefore, the use of a differential calculus based on the maximal subalgebra of $\operatorname{Der}(\mathcal{M})$ whose elements generate infinitesimal symplectomorphisms permits one to construct naturally NC analogues of Yang-Mills-Higgs actions defined on Moyal space. Note that any attempt to interpret $\mathcal{A}_{\mu \nu}$ as possibly related to a gravitational field, owing simply to the fact that it is a symmetric tensor, would be physically misleading at least in view of the fact that the symmetry group of the Moyal space is $S O(D) \cap S P(D)$ but not $S O(D)$. 


\section{Discussion}

The derivation-based differential calculus is a mathematical algebraic framework that permits one to generate from a given associative algebra different consistent differential calculi. The case of Moyal algebras has been considered in the present paper. Let us compare this latter situation with two other noncommutative geometries, which share some common structures with the one studied here.

First consider $M_{n}(\mathbb{C})$, the finite dimensional algebra of $n \times n$ matrices. The algebra $M_{n}(\mathbb{C})$ has only inner derivations, a trivial center $\mathbb{C}$ and admits canonical NC gauge invariant connections. This last property is insured by the existence in each case of a $\mathbb{C}$-linear map $\eta: \operatorname{Der}(\mathbb{A}) \rightarrow \mathbb{A}$ such that $X(a)=[\eta(X), a]$ for any $a \in \mathbb{A}\left(\mathbb{A}=M_{n}(\mathbb{C})\right.$ or $\left.\mathcal{M}\right)$. This map defines a canonical gauge invariant connection on (the right $\mathbb{A}$-module) $\mathbb{A}: a \mapsto \nabla_{X} a=-a \eta(X)$. For the differential calculus based on a subalgebra of $\operatorname{Der}(\mathcal{M})$, as considered here, the map $\eta$ is defined by (3.6). For the differential calculus based on $\operatorname{Der}\left(M_{n}(\mathbb{C})\right)$, the map is defined by the canonical 1-form connection $i \theta$ of $M_{n}(\mathbb{C}), i \theta \in \Omega_{\text {Der }}^{1}\left(M_{n}(\mathbb{C})\right)$ interpreted as a map $\operatorname{Int}\left(M_{n}(\mathbb{C})\right) \rightarrow M_{n}(\mathbb{C})$ and such that $i \theta\left(\operatorname{Ad}_{\gamma}\right)=\gamma-\frac{1}{n} \operatorname{Tr}(\gamma) \mathbb{I}, \forall \gamma \in M_{n}(\mathbb{C})$. However, this last map is a morphism of Lie algebra from $\operatorname{Der}\left(M_{n}(\mathbb{C})\right)$ to the subalgebra of $M_{n}(\mathbb{C}$ formed by the traceless elements (the Lie bracket is the usual commutator) and therefore, the curvature of the canonical connection is zero. This is not the case for the differential calculus considered here. The map $\eta$ defined in (3.6) is not a Lie algebra morphism which is signaled by a non zero curvature for the canonical connection.

Consider now the algebra $\mathbb{A}=C^{\infty}(M) \otimes M_{n}(\mathbb{C})$ of matrix valued functions on a smooth finite dimensional manifold $M$ whose derivation-based differential calculus was first considered in [37]. In the present case, $\mathcal{Z}(\mathbb{A})=C^{\infty}(M)$ and $\operatorname{Der}(\mathbb{A})=\left[\operatorname{Der}\left(C^{\infty}(M)\right) \otimes \mathbf{1}\right] \oplus\left[C^{\infty}(M) \otimes \operatorname{Der}\left(M_{n}\right)\right]=$ $\Gamma(M) \oplus\left[C^{\infty}(M) \otimes \mathfrak{s l}_{n}\right]$ in the sense of Lie algebras and $C^{\infty}(M)$-modules. $\Gamma(M)$ is the Lie algebra of smooth vector fields on $M$. Then, for any derivation $\mathcal{X} \in \operatorname{Der}(\mathbb{A}), \mathcal{X}=X+a d_{\gamma}$ with $X \in \Gamma(M)$ and $\gamma \in C^{\infty}(M) \otimes \mathfrak{s l}_{n}$, the traceless elements in $\mathbb{A}$. Set $\mathbb{A}_{0}=C^{\infty}(M) \otimes \mathfrak{s l}$. One can identify $\operatorname{Int}(\mathbb{A})=\mathbb{A}_{0}$ and $\operatorname{Out}(\mathbb{A})=\Gamma(M)$. Therefore, one has both inner and outer derivations. Finally, one has $\Omega_{\text {Der }}^{*}(\mathbb{A})=\Omega^{*}(M) \otimes \Omega_{\text {Der }}^{*}\left(M_{n}\right)$ with a differential $\hat{\mathrm{d}}=\mathrm{d}+\mathrm{d}^{\prime}$, where $\mathrm{d}$ is the de Rham differential and $\mathrm{d}^{\prime}$ is the differential operating on the $M_{n}(\mathbb{C})$ part. The 1-form related to the canonical connection is defined by $i \theta(\mathcal{X})=\gamma$. As a map from $\operatorname{Der}(\mathbb{A})$ to $\mathbb{A}_{0}$, it defines a splitting of Lie algebras and $C^{\infty}(M)$-modules of the short exact sequence

$$
0 \longrightarrow \mathbb{A}_{0} \longrightarrow \operatorname{Der}(\mathbb{A}) \longrightarrow \operatorname{Out}(\mathbb{A}) \longrightarrow 0
$$

while the map $\eta$ defined by (3.6) does not have a similar property. The canonical connection on (the right $\mathbb{A}$-module) $\mathbb{A}$ is defined from $-i \theta$ by $\nabla_{\mathcal{X}}(a)=\mathcal{X}(a)-i \theta(\mathcal{X}) a=X(a)-a \gamma, \forall a \in \mathbb{A}$ but is not gauge invariant while the corresponding curvature is zero, due to the above property of splitting of Lie algebras. Past classical studies of the corresponding gauge theories, with an action constructed mainly from the square of the curvature, gave rise to the interpretation of the gauge potential as involving two parts, one being the "ordinary" gauge theories and the other one identifiable as Higgs fields. Indeed, one can show that the simple action $\sim \int d^{D} x F_{\mu \nu} F^{\mu \nu}$ constructed using the corresponding curvature $F_{\mu \nu}$ exhibits non trivial vacuum states in the Higgs part, from which a mass generation on the "ordinary" gauge fields is a consequence. This situation has been generalised [38, 39] to the case of the algebra of endomorphisms of a $S U(n)$ vector bundle in the sense that the situation of the trivial bundle correspond to the algebra of matrix-valued functions. Because of the possible non trivial global topology of the bundle, the situation is more involved [38] but reveals essentially that this physical interpretation of the components of the noncommutative gauge fields can be performed in the same way.

The Yang-Mills-Higgs type action constructed from differential calculus based on the subalgebra $\mathcal{G}_{2} \subset \operatorname{Der}(\mathcal{M})$ in Section 3 shares common features with this last situation: Each additional inner derivation supplementing the "ordinary spatial derivations", which may be viewed as re- 
lated to an extra noncommutative dimension, corresponds to an additional covariant coordinate that can be interpreted as a Higgs field. Covariant coordinates have thus a natural interpretation as Higgs fields within the framework of the present derivation-based differential calculus. Then, Yang-Mills-Higgs models can be obtained from actions built from the square of the curvature (3.13)-(3.15).

In these type of models, the polarisation tensor for the gauge potential $A_{\mu}$ still exhibits an IR singularity similar to the one given in (1.1) with however a different overall factor depending in particular on the dimension $D$ of the noncommutative space and the Higgs fields content. The calculation can be performed by using auxiliary integrals given below. We consider here the case where no bare mass term for the gauge potential is present. Inclusion of a bare mass term would not alter the conclusion. It is convenient to set $\Phi_{a} \equiv \mathcal{A}_{\mu \nu}, a=1, \ldots, \frac{D(D+1)}{2}$ and parametrise the gauge invariant action as (we define $p \wedge k \equiv p_{\mu} \Theta_{\mu \nu} k_{\nu}$ )

$$
S_{c l}=\int d^{D} x \frac{1}{4}\left(F_{\mu \nu} \star F_{\mu \nu}+\left(D_{\mu} \Phi_{a}\right)^{2}+F_{a b} \star F_{a b}\right),
$$

where the coupling constant $\alpha$ has been set equal to 1 and $D_{\mu} \Phi_{a}=\left[\mathcal{A}_{\mu}, \Phi_{a}\right]_{\star}=\partial_{\mu} \Phi_{a}-i\left[A_{\mu}, \Phi_{a}\right]_{\star}$. The action $S_{c l}$ must be supplemented by a BRST-invariant gauge fixing term $S_{G F}$, which can be taken as

$$
S_{G F}=s \int d^{D} x\left(\bar{C} \partial^{\mu} A_{\mu}+\frac{\lambda}{2} \bar{C} b\right)=\int d^{D} x\left(b \partial^{\mu} A_{\mu}-\bar{C} \partial^{\mu}\left(\partial_{\mu} C-i\left[A_{\mu}, C\right]_{\star}\right)+\frac{\lambda}{2} b^{2}\right),
$$

where the nilpotent Slavnov operation $s$ is defined through the following BRST structure equations

$$
s A_{\mu}=\partial_{\mu} C-i\left[A_{\mu}, C\right]_{\star}, \quad s C=i C \star C, \quad s \bar{C}=b, \quad s b=0,
$$

where $\lambda$ is a real constant and $C, \bar{C}$ and $b$ denote respectively the ghost, the antighost and the Stuekelberg auxiliary field with ghost number respectively equal to $+1,-1$ and 0 . Recall that $s$ acts as a graded derivation on the various objects with grading defined by the sum of the degree of differential forms and ghost number (modulo 2). In the following, we will perform the calculation using a Feynman-type gauge. Accordingly, the propagator for the $A_{\mu}$ in momentum space takes the diagonal form $G_{\mu \nu}(p)=\delta_{\mu \nu} / p^{2}$. The ghost and Higgs propagators are respectively given by $G_{g}(p)=1 / p^{2}$ and $G_{a b}^{H}(p)=2 \delta_{a b} /\left(p^{2}+\mu^{2}\right)$. The Feynman rules used in the course of the calculation are given in the Appendix A.

The one-loop diagrams contributing to the vacuum polarisation tensor $\omega_{\mu \nu}(p)$ are depicted on the Fig. 1. The respective contributions can be written as

$$
\begin{aligned}
\omega_{\mu \nu}^{1}(p)= & 4 \int \frac{d^{D} k}{(2 \pi)^{D}} \frac{\sin ^{2}\left(\frac{p \wedge k}{2}\right)}{k^{2}(p+k)^{2}}\left[\left((k-p)^{2}+(k+2 p)^{2}\right) \delta_{\mu \nu}+(D-6) p_{\mu} p_{\nu}\right. \\
& \left.+\left(p_{\mu} k_{\nu}+k_{\mu} p_{\nu}\right)(2 D-3)+k_{\mu} k_{\nu}(4 D-6)\right], \\
\omega_{\mu \nu}^{2}(p)= & 4 \int \frac{d^{D} k}{(2 \pi)^{D}} \frac{\sin ^{2}\left(\frac{p \wedge k}{2}\right)}{k^{2}(p+k)^{2}} k_{\mu} k_{\nu}, \quad \omega_{\mu \nu}^{3}(p)=8(D-1) \delta_{\mu \nu} \int \frac{d^{D} k}{(2 \pi)^{D}} \frac{\sin ^{2}\left(\frac{p \wedge k}{2}\right)}{k^{2}}, \\
\omega_{\mu \nu}^{4}(p)= & 4 \mathcal{N} \int \frac{d^{D} k}{(2 \pi)^{D}} \frac{\sin ^{2}\left(\frac{p \wedge k}{2}\right)}{\left(k^{2}+\mu^{2}\right)\left((p+k)^{2}+\mu^{2}\right)}(p+2 k)_{\mu}(p+2 k)_{\nu}, \\
\omega_{\mu \nu}^{5}(p)= & -4 \mathcal{N} \delta_{\mu \nu} \int \frac{d^{D} k}{(2 \pi)^{D}} \frac{\sin ^{2}\left(\frac{p \wedge k}{2}\right)}{\left(k^{2}+\mu^{2}\right)}
\end{aligned}
$$

where $\mathcal{N}$ is the number of $\Phi$ fields, i.e. $\mathcal{N}=\frac{D(D+1)}{2}$ for $\operatorname{sp}(D, \mathbb{R})$. 

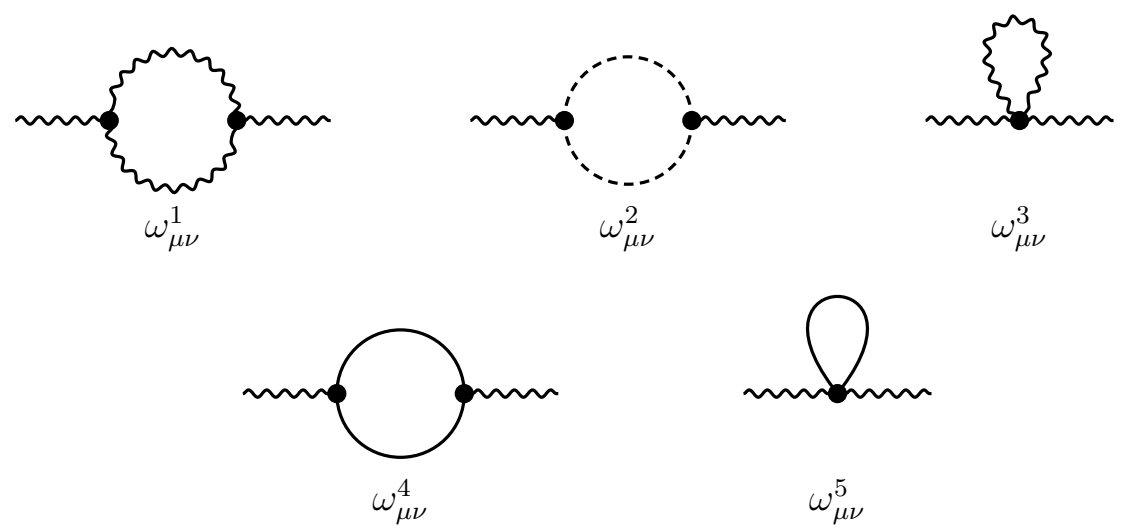

$\omega_{\mu \nu}^{5}$

Figure 1. One-loop diagrams contributing to the vacuum polarisation tensor. The wavy lines correspond to $A_{\mu}$. The full (resp. dashed) lines correspond to the $\Phi$ (resp. ghost) fields.

It is convenient here to use the Feynman parametrisation, namely $\frac{1}{a b}=\int_{0}^{1} d x \frac{1}{(x a+(1-x) b)^{2}}$. Then, standard manipulations permit one to extract the IR limit of (4.1)-(4.4), denoted by $\omega_{\mu \nu}^{\mathrm{IR}}(p)$. In the course of the derivation, it is very convenient to use the following integrals

$$
\begin{aligned}
& J_{N}(\tilde{p}) \equiv \int \frac{d^{D} k}{(2 \pi)^{D}} \frac{e^{i k \tilde{p}}}{\left(k^{2}+m^{2}\right)^{N}}=a_{N, D} \mathcal{M}_{N-\frac{D}{2}}(m|\tilde{p}|), \\
& J_{N, \mu \nu}(\tilde{p}) \equiv \int \frac{d^{D} k}{(2 \pi)^{D}} \frac{k_{\mu} k_{\nu} e^{i k \tilde{p}}}{\left(k^{2}+m^{2}\right)^{N}}=a_{N, D}\left(\delta_{\mu \nu} \mathcal{M}_{N-1-\frac{D}{2}}(m|\tilde{p}|)-\tilde{p}_{\mu} \tilde{p}_{\nu} \mathcal{M}_{N-2-\frac{D}{2}}(m|\tilde{p}|)\right),
\end{aligned}
$$

where

$$
a_{N, D}=\frac{2^{-\left(\frac{D}{2}+N-1\right)}}{\Gamma(N) \pi^{\frac{D}{2}}}, \quad \mathcal{M}_{Q}(m|\tilde{p}|)=\frac{1}{\left(m^{2}\right)^{Q}}(m|\tilde{p}|)^{Q} \mathbf{K}_{Q}(m|\tilde{p}|)
$$

in which $\mathbf{K}_{Q}(z)$ is the modified Bessel function of second kind and of order $Q \in \mathbb{Z}$ (recall $\left.\mathbf{K}_{-Q}(z)=\mathbf{K}_{Q}(z)\right)$ together with the asymptotic expansion

$$
\mathcal{M}_{-Q}(m|\tilde{p}|) \sim 2^{Q-1} \frac{\Gamma(Q)}{\tilde{p}^{2 Q}}, \quad Q>0 .
$$

The IR limit of the vacuum polarisation tensor is given by

$$
\omega_{\mu \nu}^{\mathrm{IR}}(p)=(D+\mathcal{N}-2) \Gamma\left(\frac{D}{2}\right) \frac{\tilde{p}_{\mu} \tilde{p}_{\nu}}{\pi^{D / 2}\left(\tilde{p}^{2}\right)^{D / 2}}+\cdots
$$

where the ellipses denote subleading singular terms. The overall factor affecting $\omega_{\mu \nu}^{\mathrm{IR}}(p)$ is modified compared to (1.1). It cannot be canceled by tuning the values for $D$ and $\mathcal{N}$. A similar comment applies to $\Pi_{a b}^{\mathrm{IR}}(p)$. Note that a one-loop calculation of the polarisation tensor performed within some $N=1$ supersymmetric version of the NC Yang-Mills theory [48] (see also [49]) suggests a better IR behaviour due basically to some compensation occurring between bosonic and fermionic loop contributions.

\section{Graded differential calculus and gauge theories}

In this section, we will construct a gauge theory starting from a $\mathbb{Z}_{2}$-graded version of the Moyal algebra as a direct application of the framework for the $\mathbb{Z}_{2}$-graded differential calculus introduced in Section 2.3. 
Consider the $\mathbb{Z}_{2}$-graded vector space $\mathbb{A}^{\bullet}=\mathcal{M}^{0} \oplus \mathcal{M}^{1}$ built from 2 copies of the Moyal algebra. $\mathcal{M}^{0}$ (resp. $\mathcal{M}^{1}$ ) involves elements of homogeneous degree 0 (resp. 1). We denote any element $a \in \mathbb{A}^{\bullet}$ by $a=\left(a_{0}, a_{1}\right), a_{i} \in \mathcal{M}^{i}, i=1,2$.

Proposition 5.1. $\mathbb{A}^{\bullet}=\mathcal{M}^{0} \oplus \mathcal{M}^{1}$ equipped with the internal product

$$
a b=\left(a_{0} \star b_{0}+a_{1} \star b_{1}, a_{0} \star b_{1}+a_{1} \star b_{0}\right), \quad \forall a=\left(a_{0}, a_{1}\right), b=\left(b_{0}, b_{1}\right) \in \mathcal{A}^{\bullet},
$$

where $\star$ is the Moyal product and the involution $a^{\dagger}=\left(a_{0}^{\dagger}, i a_{1}^{\dagger}\right)$ where $a_{i}^{\dagger}, i=0,1$ is the natural involution on the Moyal algebra, is an associative $*$-algebra wit unit $\mathbb{I}=(1,0)$. The center is $\mathcal{Z}\left(\mathbb{A}^{\bullet}\right)=\mathbb{C} \oplus 0$.

Proof. Each part of the proposition can be proved by simple calculation. Note that the determination of the center stems from $\mathcal{Z}(\mathcal{M})=\mathbb{C}$.

An interesting generalisation of the NC gauge theory constructed thorough Sections 3.2 and 3.3 to a NC gauge theory defined on the $\mathbb{Z}_{2}$-graded algebra $\mathcal{A}^{\bullet}$ can be obtained as follows. We define

$$
\begin{aligned}
& T_{\mu}=\left(\eta_{\mu}, 0\right), \quad U_{\mu}=\left(0, \eta_{\mu}\right), \quad M_{\mu \nu}=\left(\eta_{\mu \nu}, 0\right), \\
& J=(0, i), \quad \eta_{\mu}=i \xi_{\mu}, \quad \eta_{\mu \nu}=i \xi_{\mu} \xi_{\nu}, \quad \mu, \nu=1, \ldots, D .
\end{aligned}
$$

A useful relation can be easily shown

$$
[a, b] \bullet=\left[\left(a_{0}, a_{1}\right),\left(b_{0}, b_{1}\right)\right]_{\bullet}=\left(\left[a_{0}, b_{0}\right]_{\star}+\left\{a_{1}, b_{1}\right\}_{\star},\left[a_{0}, b_{1}\right]_{\star}+\left[a_{1}, b_{0}\right]_{\star}\right), \quad \forall a, b \in \mathbb{A}^{\bullet} .
$$

First, consider the Lie subalgebra of $\operatorname{Der}\left(\mathbb{A}^{\bullet}\right)$ generated by the real derivations of degree $0 \operatorname{Ad}_{T_{\mu}}$ and $\operatorname{Ad}_{M_{\mu \nu}}$, isomorphic to $\mathcal{G}_{2} \subset \operatorname{Der}\left(\mathcal{M}^{0}\right)$ defined in Section 3.2. Then, enlarge this subalgebra by adding the real derivation of degree $1 \operatorname{Ad}_{J}$ and $\operatorname{Ad}_{U_{\mu}}$. Notice that $J$ does not belong to $\mathcal{Z}\left(\mathbb{A}^{\bullet}\right)$ so that $\operatorname{Ad}_{J}$ is not the trivial 0 derivation. One has the following commutation relations

$$
\begin{aligned}
& {\left[T_{\mu}, T_{\nu}\right]_{\bullet}=i \Theta_{\mu \nu}^{-1} \mathbb{I}, \quad\left[M_{\mu \nu}, T_{\rho}\right]_{\bullet}=\left(\Theta_{\nu \rho}^{-1} T_{\mu}+\Theta_{\mu \rho}^{-1} T_{\nu}\right),} \\
& {\left[M_{\mu \nu}, M_{\rho \sigma}\right]_{\bullet}=\left(\Theta_{\nu \sigma}^{-1} M_{\mu \rho}+\Theta_{\nu \rho}^{-1} M_{\mu \sigma}+\Theta_{\mu \sigma}^{-1} M_{\nu \rho}+\Theta_{\mu \rho}^{-1} M_{\nu \sigma}\right),} \\
& {\left[U_{\mu}, U_{\nu}\right]_{\bullet}=i 2 M_{\mu \nu}, \quad\left[T_{\mu}, U_{\nu}\right]_{\bullet}=\Theta_{\mu \nu}^{-1} J, \quad\left[M_{\mu \nu}, U_{\rho}\right]_{\bullet}=\left(\Theta_{\nu \rho}^{-1} U_{\mu}+\Theta_{\mu \rho}^{-1} U_{\nu}\right),} \\
& {[J, J]_{\bullet}=-2 \mathbb{I}, \quad\left[T_{\mu}, J\right]_{\bullet}=0, \quad\left[M_{\mu \nu}, J\right]_{\bullet}=0, \quad\left[U_{\mu}, J\right]_{\bullet}=i 2 T_{\mu} .}
\end{aligned}
$$

Proposition 5.2. The set of real derivations $\left\{\operatorname{Ad}_{a}\right\}, a=T_{\mu}, U_{\mu}, M_{\mu \nu}, J$ is a $\mathbb{Z}_{2}$-graded Lie subalgebra of $\operatorname{Der}\left(\mathbb{A}^{\bullet}\right)$ and module over $\mathcal{Z}\left(\mathbb{A}^{\bullet}\right)$.

Proof. The proposition is a direct consequence of the usual relation $\left[\operatorname{Ad}_{X}, \operatorname{Ad}_{Y}\right]_{\bullet}=\operatorname{Ad}_{[X, Y]}$. combined with equations (5.1)-(5.4). The properties of module are obvious.

The map $\eta$ defined in Section 3.2 can be extended as follows. One considers $\mathcal{G}_{2}^{\bullet}$, the image by the map Ad of $\left(\mathcal{P}_{2}^{0} \oplus \mathcal{P}_{1}^{1}\right) \subset\left(\mathcal{M}^{0} \oplus \mathcal{M}^{1}\right)$ where $\mathcal{P}_{2}^{0} \subset \mathcal{M}^{0}$ (resp. $\left.\mathcal{P}_{1}^{1} \subset \mathcal{M}^{1}\right)$ is the set of polynomial with degree $d \leq 2$ (resp. $d \leq 1$ ). Then, the linear map $\eta$ of Section 3 can be extended to linear map of homogeneous degree $0 \eta: \mathcal{G}_{2}^{\bullet} \rightarrow \mathbb{A}^{\bullet} / \eta(X)=\eta\left(\operatorname{Ad}_{\left(P_{0}, P_{1}\right)}=\left(P_{0}-P_{0}(0), P_{1}\right)\right.$, $P_{0} \in\left(\mathcal{P}_{2}^{0}, P_{1} \in \mathcal{P}_{1}^{1}\right.$.

We can now apply the algebraic scheme presented in Section 2.3. The components of the 1-form $\eta$ are

$$
\eta\left(\operatorname{Ad}_{T_{\mu}}\right)=\left(\eta_{\mu}, 0\right), \quad \eta\left(\operatorname{Ad}_{U_{\mu}}\right)=\left(0, \eta_{\mu}\right), \quad \eta\left(\operatorname{Ad}_{M_{\mu \nu}}\right)=\left(\eta_{\mu \nu}, 0\right), \quad \eta\left(\operatorname{Ad}_{J}\right)=(0, i) .
$$


For any $a=\left(a_{0}, a_{1}\right) \in \mathbb{A}^{\bullet}$, the 1 -form canonical connection on $\mathbb{A}^{\bullet}$ is determined by

$$
\begin{aligned}
& \nabla_{\mathrm{Ad}_{T_{\mu}}}^{\mathrm{inv}}\left(a_{0}, 0\right)=-\left(a_{0} \star \eta_{\mu}, 0\right), \quad \nabla_{\mathrm{Ad}_{T_{\mu}}}^{\mathrm{inv}}\left(0, a_{1}\right)=-\left(0, a_{1} \star \eta_{\mu}\right), \\
& \nabla_{\mathrm{Ad}_{U_{\mu}}}^{\mathrm{inv}}\left(a_{0}, 0\right)=-\left(0, a_{0} \star \eta_{\mu}\right), \quad \nabla_{\mathrm{Ad}_{T_{\mu}}}^{\mathrm{inv}}\left(0, a_{1}\right)=\left(a_{1} \star \eta_{\mu}, 0\right), \\
& \nabla_{\operatorname{Ad}_{M} \nu}^{\operatorname{inv}}\left(a_{0}, 0\right)=-\left(a_{0} \star \eta_{\mu \nu}, 0\right), \quad \nabla_{\operatorname{Ad}_{M \mu \nu}}^{\operatorname{inv}}\left(0, a_{1}\right)=-\left(0, a_{1} \star \eta_{\mu \nu}\right) \text {, } \\
& \nabla_{\mathrm{Ad}_{J}}^{\mathrm{inv}}\left(\left(a_{0}, 0\right)\right)=-\left(0, i a_{0}\right), \quad \nabla_{\mathrm{Ad}_{J}}^{\mathrm{inv}}\left(0, a_{1}\right)=\left(i a_{1}, 0\right) .
\end{aligned}
$$

We now perform the rescaling $A(X) \rightarrow-i A(X)$ as in Section 3. Then, for any NC connection on $\mathbb{A}^{\bullet}$ determined by the 1 -form $A \in \Omega_{\mathrm{Der}}^{1,0}\left(\mathbb{A}^{\bullet}\right)$, the components of the tensor 1 -form are given by

$$
\begin{aligned}
& \mathcal{A}\left(\operatorname{Ad}_{T_{\mu}}\right)=-i\left(A_{\mu}^{0}-\xi_{\mu}, 0\right) \equiv-i\left(\mathcal{A}_{\mu}^{0}, 0\right), \\
& \mathcal{A}\left(\operatorname{Ad}_{U_{\mu}}\right)=-i\left(0, A_{\mu}^{1}-\xi_{\mu}\right) \equiv-i\left(0, \mathcal{A}_{\mu}^{1}\right), \\
& \mathcal{A}\left(\operatorname{Ad}_{M_{\mu \nu}}\right)=-i\left(G_{\mu \nu}^{0}-\xi_{\mu} \xi_{\nu}, 0\right) \equiv-i\left(\mathcal{G}_{\mu \nu}^{0}, 0\right), \\
& \mathcal{A}\left(\operatorname{Ad}_{J}\right)=-i(0, \varphi-1) \equiv-i(0, \Phi),
\end{aligned}
$$

where we have defined $A\left(\operatorname{Ad}_{T_{\mu}}\right) \equiv\left(A_{\mu}^{0}, 0\right), A\left(\operatorname{Ad}_{U_{\mu}}\right) \equiv\left(0, A_{\mu}^{1}\right), A\left(\operatorname{Ad}_{M_{\mu} \nu}\right) \equiv\left(G_{\mu \nu}^{0}, 0\right)$ and $A\left(\operatorname{Ad}_{J}\right) \equiv(0, \varphi)$.

Proposition 5.3. Consider the restricted differential calculus based on $\mathcal{G}_{2}^{\bullet} \subset \operatorname{Der}\left(\mathbb{A}^{\bullet}\right)$ given in Proposition 5.2. The components of the 2-form curvature for a $N C$ connection on $\mathbb{A}^{\bullet}$ are:

$$
\begin{aligned}
& F\left(\operatorname{Ad}_{T_{\mu}}, \operatorname{Ad}_{T_{\nu}}\right)=\left(-\left[\mathcal{A}_{\mu}^{0}, \mathcal{A}_{\nu}^{0}\right]_{\star}-i \Theta_{\mu \nu}^{-1}, 0\right) \\
& F\left(\operatorname{Ad}_{U_{\mu}}, \operatorname{Ad}_{U_{\nu}}\right)=\left(-\left\{\mathcal{A}_{\mu}^{1}, \mathcal{A}_{\nu}^{1}\right\}_{\star}-2 \mathcal{G}_{\mu \nu}^{0}, 0\right) \\
& F\left(\operatorname{Ad}_{J}, \operatorname{Ad}_{J}\right)=(-2 \Phi \star \Phi+2,0), \quad F\left(\operatorname{Ad}_{T_{\mu}}, \operatorname{Ad}_{J}\right)=\left(0,-\left[\mathcal{A}_{\mu}^{0}, \Phi\right]_{\star}\right) \\
& F\left(\operatorname{Ad}_{U_{\mu}}, \operatorname{Ad}_{J}\right)=\left(-\left\{\mathcal{A}_{\mu}^{1}, \Phi\right\}_{\star}-2 \mathcal{A}_{\mu}^{0}, 0\right), \quad F\left(\operatorname{Ad}_{M_{\mu \nu}}, \operatorname{Ad}_{J}\right)=\left(0,-\left[\mathcal{G}_{\mu \nu}^{0}, \Phi\right]_{\star}\right), \\
& F\left(\operatorname{Ad}_{T_{\mu}}, \operatorname{Ad}_{U_{\nu}}\right)=\left(0,-\left[\mathcal{A}_{\mu}^{0}, \mathcal{A}_{\nu}^{1}\right]_{\star}+i \Theta_{\mu \nu}^{-1} \Phi\right), \\
& F\left(\operatorname{Ad}_{M_{\mu \nu}}, \operatorname{Ad}_{T_{\rho}}\right)=\left(-\left[\mathcal{G}_{\mu \nu}^{0}, \mathcal{A}_{\rho}^{0}\right]_{\star}+i \Theta_{\nu \rho}^{-1} \mathcal{A}_{\mu}^{0}+i \Theta_{\mu \rho}^{-1} \mathcal{A}_{\nu}^{0}, 0\right) \\
& F\left(\operatorname{Ad}_{M_{\mu \nu}}, \operatorname{Ad}_{U_{\rho}}\right)=\left(0,-\left[\mathcal{G}_{\mu \nu}^{0}, \mathcal{A}_{\rho}^{1}\right]_{\star}+i \Theta_{\nu \rho}^{-1} \mathcal{A}_{\mu}^{1}+i \Theta_{\mu \rho}^{-1} \mathcal{A}_{\nu}^{1}\right), \\
& F\left(\operatorname{Ad}_{M_{\mu \nu}}, \operatorname{Ad}_{M_{\rho \sigma}}\right)=\left(-\left[\mathcal{G}_{\mu \nu}^{0}, \mathcal{G}_{\rho \sigma}^{0}\right]_{\star}+i\left(\Theta_{\nu \sigma}^{-1} \mathcal{G}_{\mu \rho}^{0}+\Theta_{\nu \rho}^{-1} \mathcal{G}_{\mu \sigma}^{0}+\Theta_{\mu \sigma}^{-1} \mathcal{G}_{\nu \rho}^{0}+\Theta_{\mu \rho}^{-1} \mathcal{G}_{\nu \sigma}^{0}\right), 0\right) .
\end{aligned}
$$

Proof. The proposition can obtained by direct calculations.

The gauge transformations can be obtained from Proposition 2.10. After performing the rescaling $A(X) \rightarrow-i A(X)$, one has for any $g=\left(g_{0}, 0\right) \in \mathcal{U}\left(\mathbb{A}^{\bullet}\right)$

$$
A^{g}\left(\operatorname{Ad}_{J}\right)=i g^{\dagger} \operatorname{Ad}_{J}(g)+g^{\dagger} A\left(\operatorname{Ad}_{J}\right) g=g^{\dagger} A\left(\operatorname{Ad}_{J}\right) g
$$

where the second equality stems from $g^{\dagger} \operatorname{Ad}_{J}(g)=0$ which holds since $g$ has degree 0 , which therefore transforms as a tensor form despite the fact this quantity is actually related to a 1-form connection. From (5.13), one obtains

$$
\varphi^{g}=g_{0}^{\dagger} \star \varphi \star g_{0} .
$$

The other gauge transformations have standard expressions, in particular the gauge transformations of all other components of the 1-form connection involve an inhomogeneous term.

Set now $A_{\mu}^{0}=A_{\mu}^{1}$. A gauge invariant action of the form $\operatorname{Tr}\left(|F(X, Y)|^{2}\right)$ by using (5.5)-(5.12) where we have defined $\operatorname{Tr}(a)=\int d x^{2 n} a_{0}$, for any $a=\left(a_{0}, a_{1}\right) \in \mathbb{A}^{\bullet}$ and $a_{0} \in \mathcal{M}$ such that the integral exists. It is instructive to consider the case where $\mathcal{G}_{\mu \nu}^{0}=0$ and to express the remaining 
components of the curvature in terms of the components of the 1-form connection. We set again $F_{\mu \nu}=\partial_{\mu} A_{\nu}-\partial_{\nu} A_{\mu}-i\left[A_{\mu}, A_{\nu}\right]_{\star}$. One obtains

$$
\begin{aligned}
& F\left(\operatorname{Ad}_{T_{\mu}}, \operatorname{Ad}_{T_{\nu}}\right)=\left(-i F_{\mu \nu}, 0\right), \quad F\left(\operatorname{Ad}_{U_{\mu}}, \operatorname{Ad}_{U_{\nu}}\right)=\left(-\left\{A_{\mu}-\xi_{\mu}, A_{\nu}-\xi_{\nu}\right\}_{\star}, 0\right), \\
& F\left(\operatorname{Ad}_{J}, \operatorname{Ad}_{J}\right)=(-2 \varphi \star \varphi+4 \varphi, 0), \quad F\left(\operatorname{Ad}_{T_{\mu}}, \operatorname{Ad}_{J}\right)=\left(0,-i\left(\partial_{\mu} \varphi-i\left[A_{\mu}, \varphi\right]_{\star}\right)\right), \\
& F\left(\operatorname{Ad}_{U_{\mu}}, \operatorname{Ad}_{J}\right)=\left(-\left\{A_{\mu}, \varphi\right\}_{\star}+2 \xi_{\mu} \varphi, 0\right), \quad F\left(\operatorname{Ad}_{T_{\mu}}, \operatorname{Ad}_{U_{\nu}}\right)=\left(0, i\left(\Theta_{\mu \nu}^{-1} \varphi-F_{\mu \nu}\right)\right) .
\end{aligned}
$$

To obtain the correct mass dimensions for the fields and parameters involved in the action, one performs, as in Section 3, the rescaling $J \rightarrow \frac{1}{m \theta}, \eta_{\mu \nu} \rightarrow \mu \theta \eta_{\mu \nu}$ where the mass dimensions of $\mu$ and $m$ are $[m]=[\mu]=1$. The mass dimensions for the fields are $\left[A_{\mu}\right]=[\varphi]=\left[G_{\mu \nu}\right]=1$. $F\left(\operatorname{Ad}_{J}, \operatorname{Ad}_{J}\right)$ is modified as

$$
F\left(\operatorname{Ad}_{J}, \operatorname{Ad}_{J}\right)=\left(-2 \varphi \star \varphi+\frac{4}{m \theta} \varphi, 0\right)
$$

while the other are unchanged. Then, the gauge invariant action is

$$
\begin{aligned}
& S\left(A_{\mu}, \varphi ; \mathcal{G}_{\mu \nu}=0\right)=\frac{1}{\alpha^{2}} \int d^{2 n} x\left(F_{\mu \nu}^{2}+\left\{\mathcal{A}_{\mu}, \mathcal{A}_{\nu}\right\}^{2}\right)+\left(\Theta_{\mu \nu}^{-1} \varphi-F_{\mu \nu}\right)^{2} \\
& +\left(\partial_{\mu} \varphi-i\left[A_{\mu}, \varphi\right]_{\star}\right)^{2}+\left(\left\{A_{\mu}, \varphi\right\}_{\star}-2 \xi_{\mu} \varphi\right)^{2}+\left(4 \varphi \star \varphi \star \varphi \star \varphi-\frac{8}{m \theta} \varphi \star \varphi \star \varphi+\frac{16}{m^{2} \theta^{2}} \varphi^{2}\right) .
\end{aligned}
$$

The first two terms between parenthesis give rise to an action of the form $(1.2)$ of $[26,27]$ which has been proposed as the gauge counterpart of the renormalisable $\mathrm{NC} \varphi^{4}$ model with harmonic term [12]. However, this action is supplemented by a Slavnov term [50] of the form $\sim \int d^{2 n} x \Theta_{\mu \nu}^{-1} \varphi F_{\mu \nu}$ which is reminiscent of the so called $B F$ term (see e.g. $[52,51]$ ). The last 3 terms in (5.14) describe an action for the renormalisable $\mathrm{NC} \varphi^{4}$ model with harmonic term (supplemented however with a $\varphi^{\star 3}$ potential term) coupled in a gauge invariant way to $A_{\mu}$. The addition of a Slavnov term to the simplest NC analogue of Yang-Mills action $\int d^{2 n} x F_{\mu \nu} \star F_{\mu \nu}$ is know to lead to an action with a somewhat improved IR behaviour but does not prevent the UV/IR mixing to occur in the gauge-fixed action. Notice that in the present situation (5.14), the field $\varphi$ propagates while in [50] $\varphi$ serves as a simple multiplier enforcing a zero-curvature constraint. The fact that the NC $\varphi^{4}$ model with harmonic term appearing as a part of a gauge action $\sim \operatorname{Tr}\left(|F(X, Y)|^{2}\right)$ is a consequence of our choice for the $\mathbb{Z}_{2}$-graded algebra as well as of the particular algebra of graded derivations generating the differential calculus. However, encoding both the gauge action (1.2) and the $\mathrm{NC} \varphi^{4}$ harmonic model within a single gauge action $\operatorname{Tr}\left(|F(X, Y)|^{2}\right)$, and in particular taking advantage of the explicit gauge invariance, may prove useful to understand new features of the Langmann-Szabo duality which plays an important role in the renormalisability of this $\mathrm{NC} \varphi^{4}$ and of its actual gauge counterpart (if any). To do this, one has to consider the action with $\mathcal{G}_{\mu \nu} \neq 0$ which can be viewed again as a Yang-Mills-Higgs action with $\mathcal{G}_{\mu \nu}$ possibly interpretable as a Higgs field. Note finally that starting with actions $\sim \operatorname{Tr}\left(|F(X, Y)|^{2}\right)$ permits one to deal with simple vacua.

\section{Summary}

This section summarises the main features and results of the discussion. First, a general way to construct gauge models from the algebraic scheme presented in Section 2 is as follows. Consider $\mathbb{A}$ as a module over itself and pick some Lie subalgebra of derivations of $\mathbb{A}, \mathcal{G} \subset \operatorname{Der}(\mathbb{A})$, which is also a module over the center of $\mathbb{A}$. Then, a differential calculus can be defined from Propositions 2.2, 2.3 or whenever $\mathbb{A}$ is $\mathbb{Z}_{2}$-graded from Proposition 2.8. Correspondingly, NC connections and curvatures can then be defined as given in Definition 2.2 and Proposition 2.5 
in the non-graded case or, when $\mathbb{A}$ is $\mathbb{Z}_{2}$-graded, from Definition 2.7. Then, gauge actions can be obtained by considering the "square" of the curvatures. This general construction is applied first to the Moyal algebra, as discussed in Sections 3 and 4 , and to a $\mathbb{Z}_{2}$-graded associative algebra built from two copies of the Moyal algebra.

These two different situations have common features. In each case, the essential properties of the gauge models stem from the existence of a $\mathbb{C}$-linear map $\eta: \mathcal{G} \rightarrow \mathbb{A}$. Then, since all the considered $\mathcal{G}$ 's involve only inner derivations, Proposition 2.6 (or Lemma 2.1 in the $\mathbb{Z}_{2}$-graded case) ensures the existence of a canonical connection which turns out to be invariant under the gauge transformations. From this follows a natural construction of tensor forms, i.e. the so-called covariant coordinates, that will play the role of Higgs fields in the gauge actions. More physically, the additional derivations supplementing the ordinary "spatial derivations" that are involved in the Lie subalgebras of derivations considered below may be interpreted as related to NC directions associated with the Higgs fields.

In the non graded case presented in Sections 3 and $4, \mathbb{A}=\mathcal{M}$ and we consider the differential calculus based on the maximal Lie subalgebra $\mathcal{G}_{2} \subset \operatorname{Der}(\mathcal{M})$ that is related to symplectomorphisms. The resulting gauge models stemming from the above scheme can be interpreted as Yang-Mills-Higgs models on Moyal spaces, which however still suffer apparently from UV/IR mixing. These models have, in some sense, some similarity with the NC gauge models based on $\mathbb{A}=C^{\infty}(M) \otimes M_{n}(\mathbb{C})$ introduced in [37].

In the $\mathbb{Z}_{2}$-graded case where $\mathbb{A}$ is built from 2 copies of the Moyal algebra that is considered in Section 5, we consider a differential calculus based on a Lie subalgebra of $\operatorname{Der}(\mathbb{A})$ that may be viewed as a natural extension of $\mathcal{G}_{2}$. The resulting gauge models can again be interpreted as Yang-Mills-Higgs type models on this graded associative algebra. The gauge action involves now three interesting contributions. The first one is the action as given in (1.2) which has been proposed in [26] and [27] as a gauge counterpart of the renormalisable NC $\varphi^{4}$ model with harmonic term. The second contribution is a Slavnov term as the one initially introduced in [50] as a first attempt to cure the UV/IR mixing in the naive gauge theory on Moyal spaces. Its actual effect on the UV/IR mixing on the gauge action defined on $\mathbb{A}^{\bullet}=\mathcal{M}^{0} \oplus \mathcal{M}^{1}$ that we have obtained in Section 5 remains to be analysed. Notice that in the present situation, the vacuum configuration for the action is trivial so that the difficult problem to deal with a complicated vacuum (see $[26,30]$ ) is absent here. Finally, the full gauge action involves, as the third interesting contribution, the $\mathrm{NC}$ renormalisable $\varphi^{4}$ model with harmonic term (up to an additional $\phi^{3}$ interaction term), therefore exhibiting a link between this latter renormalisable $\mathrm{NC}$ scalar theory and gauge theories. In this framework, notice that the harmonic term may be interpreted as a quartic gauge potential coupling, built from the (gauge potential of the) canonical connection and the component of the gauge connection corresponding to the derivation generated by $(0, i)$ (see Section 5$)$.

A next step would be to study whether the vacuum polarisation tensor still involves an IR singularity of the type given by (1.1) which would then probe the actual effect of anticommutator term $\sim\left\{\mathcal{A}_{\mu}, \mathcal{A}_{\nu}\right\}_{\star}^{2}$ (combined to a Slavnov term) on the UV/IR mixing. This amounts to consider the full action and perform a gauge fixing, e.g. using the BRST formalism.

\section{A Relevant Feynman rules}

In the following vertex functions, momentum conservation is understood. All the momenta are incoming. We define $p \wedge k \equiv p_{\mu} \Theta_{\mu \nu} k_{\nu}$.

- 3-gauge boson vertex:

$$
V_{\alpha \beta \gamma}^{3}\left(k_{1}, k_{2}, k_{3}\right)=-i 2 \sin \left(\frac{k_{1} \wedge k_{2}}{2}\right)\left[\left(k_{2}-k_{1}\right)_{\gamma} \delta_{\alpha \beta}+\left(k_{1}-k_{3}\right)_{\beta} \delta_{\alpha \gamma}+\left(k_{3}-k_{2}\right)_{\alpha} \delta_{\beta \gamma}\right]
$$


- 4-gauge boson vertex:

$$
\begin{aligned}
V_{\alpha \beta \gamma \delta}^{4}\left(k_{1}, k_{2}, k_{3}, k_{4}\right)= & -4\left[\left(\delta_{\alpha \gamma} \delta_{\beta \delta}-\delta_{\alpha \delta} \delta_{\beta \gamma}\right) \sin \left(\frac{k_{1} \wedge k_{2}}{2}\right) \sin \left(\frac{k_{3} \wedge k_{4}}{2}\right)\right. \\
& +\left(\delta_{\alpha \beta} \delta_{\gamma \delta}-\delta_{\alpha \gamma} \delta_{\beta \delta}\right) \sin \left(\frac{k_{1} \wedge k_{4}}{2}\right) \sin \left(\frac{k_{2} \wedge k_{3}}{2}\right) \\
& \left.+\left(\delta_{\alpha \delta} \delta_{\beta \gamma}-\delta_{\alpha \beta} \delta_{\gamma \delta}\right) \sin \left(\frac{k_{3} \wedge k_{1}}{2}\right) \sin \left(\frac{k_{2} \wedge k_{4}}{2}\right)\right]
\end{aligned}
$$

- gauge boson-ghost $V_{\mu}^{g}\left(k_{1}, k_{2}, k_{3}\right)$ and gauge boson-Higgs $V_{a b \mu}^{H}\left(k_{1}, k_{2}, k_{3}\right)$ vertices:

$$
\begin{aligned}
& V_{\mu}^{g}\left(k_{1}, k_{2}, k_{3}\right)=i 2 k_{1 \mu} \sin \left(\frac{k_{2} \wedge k_{3}}{2}\right), \\
& V_{a b \mu}^{H}\left(k_{1}, k_{2}, k_{3}\right)=i \delta_{a b}\left(k_{1}-k_{2}\right)_{\mu} \sin \left(\frac{k_{2} \wedge k_{3}}{2}\right) ;
\end{aligned}
$$

- Seagull vertex:

$$
\begin{aligned}
V_{a b \alpha \beta}^{s}\left(k_{1}, k_{2}, k_{3}, k_{4}\right)= & -2 \delta_{\alpha \beta} \delta_{a b}\left[\cos \left(\frac{k_{3} \wedge k_{1}+k_{4} \wedge k_{2}}{2}\right)\right. \\
& \left.-\cos \left(\frac{k_{1} \wedge k_{2}}{2}\right) \cos \left(\frac{k_{3} \wedge k_{4}}{2}\right)\right]
\end{aligned}
$$

- 3-Higgs $V_{a b c}^{H}\left(k_{1}, k_{2}, k_{3}\right)$ vertex

$$
V_{a b c}^{H}\left(k_{1}, k_{2}, k_{3}\right)=i C_{a b}^{c} \sin \left(\frac{k_{1} \wedge k_{2}}{2}\right)
$$

- 4-Higgs vertex:

$$
\begin{aligned}
V_{a b c d}^{H}\left(k_{1}, k_{2}, k_{3}, k_{4}\right)= & 4\left[\left(\delta_{a c} \delta_{b d}-\delta_{a d} \delta_{b c}\right) \sin \left(\frac{k_{1} \wedge k_{2}}{2}\right) \sin \left(\frac{k_{3} \wedge k_{4}}{2}\right)\right. \\
& +\left(\delta_{a b} \delta_{c d}-\delta_{a c} \delta_{b d}\right) \sin \left(\frac{k_{1} \wedge k_{4}}{2}\right) \sin \left(\frac{k_{2} \wedge k_{3}}{2}\right) \\
& \left.+\left(\delta_{a d} \delta_{b c}-\delta_{a b} \delta_{c d}\right) \sin \left(\frac{k_{3} \wedge k_{1}}{2}\right) \sin \left(\frac{k_{2} \wedge k_{4}}{2}\right)\right] .
\end{aligned}
$$

\section{Acknowledgements}

It is a pleasure to thank the organisers of the XVIIth International Colloquium on Integrable Systems and Quantum Symmetries for their kind invitation. Most of the results presented in this paper have been obtained from various collaborations with E. Cagnache, A. de Goursac and T. Masson. Fruitful discussions with M. Dubois-Violette and J. Madore are gratefully acknowledged.

\section{References}

[1] Douglas M.R., Nekrasov N.A., Noncommutative field theory, Rev. Mod. Phys. 73 (2001), 977-1029, hep-th/0106048.

[2] Szabo R.J., Quantum field theory on noncommutative spaces, Phys. Rept. 378 (2003), 207-299, hep-th/0109162. 
[3] Connes A., Noncommutative geometry, Academic Press, Inc., San Diego, CA, 1994, available at http://www.alainconnes.org/downloads.html.

[4] Connes A., Marcolli M., A walk in the noncommutative garden, 2006, available at http://www. alainconnes.org/downloads.html.

[5] Schomerus V., D-branes and deformation quantization, J. High Energy Phys. 1999 (1999), no. 6, 030, 14 pages, hep-th/9903205.

[6] Seiberg N., Witten E., String theory and noncommutative geometry, J. High Energy Phys. 1999 (1999), no. 9, 032, 93 pages, hep-th/9908142.

[7] Gracia-Bondía J.M., Várilly J.C., Algebras of distributions suitable for phase space quantum mechanics. I, J. Math. Phys. 29 (1988), 869-879.

[8] Várilly J.C., Gracia-Bondía J.M., Algebras of distributions suitable for phase-space quantum mechanics. II. Topologies on the Moyal algebra, J. Math. Phys. 29 (1988), 880-887.

[9] Minwalla S., Van Raamsdonk M., Seiberg N., Noncommutative perturbative dynamics, J. High Energy Phys. 2000 (2000), no. 2, 020, 31 pages, hep-th/9912072.

[10] Chepelev I., Roiban R., Renormalization of quantum field theories on noncommutative $\mathbb{R}^{d}$. I. Scalars, J. High Energy Phys. 2000 (2000), no. 5, 037, 31 pages, hep-th/9911098.

[11] Matusis A., Susskind L., Toumbas N., The IR/UV connection in the non-commutative gauge theories, J. High Energy Phys. 2000 (2000), no. 12, 002, 18 pages, hep-th/0002075.

[12] Grosse H., Wulkenhaar R., Renormalisation of $\phi^{4}$-theory on noncommutative $\mathbb{R}^{4}$ in the matrix base, Comm. Math. Phys. 256 (2005), 305-374, hep-th/0401128.

[13] Grosse H., Wulkenhaar R., Power-counting theorem for non-local matrix models and renormalisation, Comm. Math. Phys. 254 (2005), 91-127, hep-th/0305066.

[14] Rivasseau V., Non-commutative renormalization, arXiv:0705.0705.

[15] Wallet J.C., Noncommutative induced gauge theories on Moyal spaces, J. Phys. Conf. Ser. 103 (2008), 012007, 20 pages, arXiv:0708.2471.

[16] Langmann E., Szabo R.J., Duality in scalar field theory on noncommutative phase spaces, Phys. Lett. B 533 (2002), 168-177, hep-th/0202039.

[17] Grosse H., Wulkenhaar R., Renormalisation of $\phi^{4}$-theory on noncommutative $\mathbb{R}^{2}$ in the matrix base, J. High Energy Phys. 2003 (2003), no. 12, 019, hep-th/0307017.

[18] Langmann E., Szabo R.J., Zarembo K., Exact solution of quantum field theory on noncommutative phase spaces, J. High Energy Phys. 2004 (2004), no. 1, 017, 69 pages, hep-th/0308043.

[19] Langmann E., Szabo R.J., Zarembo K., Exact solution of noncommutative field theory in background magnetic fields, Phys. Lett. B 569 (2003), 95-101, hep-th/0303082.

[20] Vignes-Tourneret F., Renormalization of the orientable non-commutative Gross-Neveu model, Ann. Henri Poincaré 8 (2007), 427-474, math-ph/0606069.

[21] Grosse H., Wulkenhaar R., The $\beta$-function in duality-covariant noncommutative $\phi^{4}$ theory, Eur. Phys. J. C Part. Fields 35 (2004), 277-282, hep-th/0402093.

[22] Lakhoua A., Vignes-Tourneret F., Wallet J.C., One-loop $\beta$-functions for the orientable non-commutative Gross-Neveu model, Eur. Phys. J. C Part. Fields 52 (2007), 735-742, hep-th/0701170.

[23] Disertori M., Gurau R., Magnen J., Rivasseau V., Vanishing of beta function of non commutative $\phi_{4}^{4}$ theory to all orders, Phys. Lett. B 649 (2007), 95-102, hep-th/0612251.

[24] Gurau R., Magnen J., Rivasseau V., Tanasa A., A translation-invariant renormalizable non-commutative scalar model, arXiv:0802.0791.

[25] Blaschke D.N., Gieres F., Kronberger E., Schweda M., Wohlgenannt M., Translation-invariant models for non-commutative gauge fields, J. Phys. A: Math. Theor. 41 (2008), 252002, 7 pages, arXiv:0804.1914.

[26] de Goursac A., Wallet J.C., Wulkenhaar R., Noncommutative induced gauge theory, Eur. Phys. J. C Part. Fields 51 (2007), 977-987, hep-th/0703075.

[27] Grosse H., Wohlgenannt M., Induced gauge theory on a noncommutative space, Eur. Phys. J. C Part. Fields 52 (2007), 435-450, hep-th/0703169.

[28] de Goursac A., On the effective action of noncommutative Yang-Mills theory, J. Phys. Conf. Ser. 103 (2008), 012010, 16 pages, arXiv:0710.1162. 
[29] Grosse H., Wohlgennant M., Noncommutative QFT and renormalization, J. Phys. Conf. Ser. 53 (2006), 764-792, hep-th/0607208.

[30] de Goursac A., Wallet J.C., Wulkenhaar R., On the vacuum states for noncommutative gauge theories, Eur. Phys. J. C Part. Fields 56 (2008), 293-304, arXiv:0803.3035.

[31] de Goursac A., Tanasa A., Wallet J.C., Vacuum configurations for renormalizable non-commutative scalar models, Eur. Phys. J. C Part. Fields 53 (2008), 459-466, arXiv:0709.3950.

[32] Dubois-Violette M., Dérivations et calcul différentiel non commutatif, C. R. Acad. Sci. Paris Sér. I Math. 307 (1988), 403-408.

[33] Dubois-Violette M., Michor P.W., Dérivations et calcul différentiel non commutatif. II, C. R. Acad. Sci. Paris Sér. I Math. 319 (1994), 927-931.

[34] Dubois-Violette M., Kerner R., Madore J., Noncommutative differential geometry of matrix algebras, J. Math. Phys. 31 (1990), 316-322.

[35] Dubois-Violette M., Michor P.W., Connections on central bimodules in noncommutative differential geometry, J. Geom. Phys. 20 (1996), 218-232, q-alg/9503020.

[36] Dubois-Violette M., Lectures on graded differential algebras and noncommutative geometry, in Proc. of the Workshop on Noncommutative Differential Geometry and Its Applications to Physics (Shonan, Japan, 1999), Editors Y. Maeda et al., Math. Phys. Stud., Vol. 23, Dordrecht, Kluwer Academic Publishers, 2001, 245-306, math.QA/9912017.

[37] Dubois-Violette M., Kerner R., Madore J., Noncommutative differential geometry and new models of gauge theory, J. Math. Phys. 31 (1990), 323-330.

[38] Dubois-Violette M., Masson T., SU(n)-connections and noncommutative differential geometry, J. Geom. Phys. 25 (1998), 104-118, dg-ga/9612017.

[39] Masson T., On the noncommutative geometry of the endomorphism algebra of a vector bundle, J. Geom. Phys. 31 (1999), 142-152, math.DG/9803088.

Masson T., Submanifolds and quotient manifolds in noncommutative geometry, J. Math. Phys. 37 (1996), 2484-2497, q-alg/9507030.

[40] Masson T., Noncommutative generalization of $S U(n)$-principal fiber bundles: a review, J. Phys. Conf. Ser. 103 (2008), 012003, 33 pages, arXiv:0709.0856.

[41] Cagnache E., Masson T., Wallet J.C., Noncommutative Yang-Mills-Higgs actions from derivation-based differential calculus, arXiv:0804.3061.

[42] Marmo G., Vitale P., Zampini A., Noncommutative differential calculus for Moyal subalgebras, J. Geom. Phys. 56 (2006), 611-622, hep-th/0411223.

[43] Scheunert M., Generalized Lie algebras, J. Math. Phys. 20 (1979), 712-720.

[44] de Goursac A., Masson T., Wallet J.C., Noncommutative $\varepsilon$-graded connections and application to Moyal space, arXiv:0811.3567.

[45] Quillen D., Superconnections and the Chern character, Topology 24 (1985), 89-95.

[46] de Goursac A., Masson T., Wallet J.C., Work in progress.

[47] Estrada R., Gracia-Bondía J.M., Várilly J.C., On asymptotic expansions of twisted products, J. Math. Phys. 30 (1989), 2789-2796.

[48] Bichl A.A., Ertl M., Gerhold A., Grimstrup J.M., Grosse H., Popp L., Putz V., Schweda M., Wulkenhaar R., Non-commutative $U(1)$ super Yang-Mills theory: Perturbative self-energy corrections, Internat. J. Modern Phys. A 19 (2004), 4231-4249, hep-th/0203141.

[49] Martin C.P., Sánchez-Ruiz D., The one-loop UV divergent structure of U(1) Yang-Mills theory on noncommutative $\mathbb{R}^{4}$, Phys. Rev. Lett. 83 (1999), 476-479, hep-th/9903077.

Grosse H., Krajewski T., Wulkenhaar R., Renormalisation of non-commutative Yang-Mills theories: a simple example, hep-th/0001182.

[50] Slavnov A.A., Consistent noncommutative quantum gauge theories, Phys. Lett. B. 565 (2003), 246-252, hep-th/0304141.

[51] Wallet J.C., Algebraic set-up for the gauge-fixing of BF and SuperBF systems, Phys. Lett. B 235 (1990), $71-78$.

[52] Birmingham D., Blau M., Rakowski M., Thomson G., Topological field theory, Phys. Rep. 209 (1991), 129-340. 